\title{
Revisiting the Low-Frequency Dipolar Perturbation by an Impenetrable Ellipsoid in a Conductive Surrounding
}

\author{
Panayiotis Vafeas \\ Department of Chemical Engineering, University of Patras, 26504 Patras, Greece \\ Correspondence should be addressed to Panayiotis Vafeas; vafeas@chemeng.upatras.gr
}

Received 27 June 2017; Accepted 11 October 2017; Published 9 November 2017

Academic Editor: Filippo Cacace

Copyright (C) 2017 Panayiotis Vafeas. This is an open access article distributed under the Creative Commons Attribution License, which permits unrestricted use, distribution, and reproduction in any medium, provided the original work is properly cited.

\begin{abstract}
This contribution deals with the scattering by a metallic ellipsoidal target, embedded in a homogeneous conductive medium, which is stimulated when a $3 \mathrm{D}$ time-harmonic magnetic dipole is operating at the low-frequency realm. The incident, the scattered, and the total three-dimensional electromagnetic fields, which satisfy Maxwell's equations, yield low-frequency expansions in terms of positive integral powers of the complex-valued wave number of the exterior medium. We preserve the static Rayleigh approximation and the first three dynamic terms, while the additional terms of minor contribution are neglected. The Maxwell-type problem is transformed into intertwined potential-type boundary value problems with impenetrable boundary conditions, whereas the environment of a genuine ellipsoidal coordinate system provides the necessary setting for tackling such problems in anisotropic space. The fields are represented via nonaxisymmetric infinite series expansions in terms of harmonic eigenfunctions, affiliated with the ellipsoidal system, obtaining analytical closed-form solutions in a compact fashion. Until nowadays, such problems were attacked by using the very few ellipsoidal harmonics exhibiting an analytical form. In the present article, we address this issue by incorporating the full series expansion of the potentials and utilizing the entire subspace of ellipsoidal harmonic eigenfunctions.
\end{abstract}

\section{Introduction}

Inductive electromagnetic means that are currently employed in several practical applications in physics, which are relative to electromagnetic activities, deal with many configurations of sources and receivers. The uncertainty resulting from datasets containing both the contribution of the primaryincident field and the secondary-scattered field explains the continuous interest of elaborating within the frame of analytical and numerical methods of solving forward and inverse electromagnetic scattering problems. In this direction, we are often faced with the problem of identifying and retrieving anomalies of a certain kind, usually behaving as perfect conductors, embedded within environment with conductive properties. The goal is to get a versatile set of mathematical and computational tools in order to infer information on the unknown body, which scatters off when it is illuminated by a known source operating nearby. The first stage of the work consists in the development of simple yet accurate models of the scattering problem itself, which can bring insight to the field behaviour and be employed at low computational cost, in view of a nonlinear inversion scheme, aiming at the retrieval of main geometrical and electrical parameters that characterize the object.

In such analytical or semianalytical approaches, we are confronted with a near-field problem, where planar skin depths are significantly larger than source-body or bodysensor distances and, therein, only diffusion phenomena occur, since conduction currents are predominant. To this end, the low-frequency electromagnetic scattering theory [1] is adopted in order to specify the kinds of the metallic targets with nondestructive analytical methods, which remains a subject of worthwhile investigation, even if there exist computational tools that could directly provide numerical data. Indeed, whenever analytical solutions are found, it is expected to obtain accurate means to check the suitability of these most probably computationally demanding solutions, as well as fast means to invert scattered field data, collected around similar bodies in order to yield crucial information about them. This is indisputable true in exploration of conductive media and possibly highly conducting embedded bodies for which the frequency range is often quite low due to its 
conductive character, meaning that low-frequency models are pertinent.

The ellipsoidal shape $[2,3]$ is highly versatile and easily matches single obstacles of smooth surface and arbitrary proportions, while such simplified geometries provide a proper first model when dealing with similar situations, where efficient mathematical tools [4] could be applied. On the other hand, the assumption of impenetrable ellipsoidal bodies is realistic in view of their high conductivity, their huge conductivity ratio with respect to the surrounding medium, and the low operation frequencies. Indeed, present investigations $[5,6]$ confirm that simple models as ours appear reliable when used to model the response of a general three-dimensional ellipsoid to a localized vector source in a homogeneous conductive medium both for low-contrast and high-contrast cases. However, the difficulty induced in performing analytical techniques when we are moving towards anisotropic geometrical models is strongly increasing due to the appearance of much more elaborate corresponding eigenfunctions of the introduced potentials, though the already rich literature with analytical works concerning the scattering by simple nonpenetrable metal shapes like spheres [7-9], spheroids [10, 11], and as already mentioned ellipsoids $[5,6]$ is open to accept new and useful analytical results. Indeed, very recently, similar analytical techniques based on differential analysis were adopted for targeting toroidal metallic objects within either a conductive surrounding, for example, Earth [12] or a lossless medium, for example, air [13]. Nevertheless, aspects dealing with integral methods stand in the frontline of the current research, for example, an inverse scheme is used to localize a smooth surface of a three-dimensional perfectly conducting object using a boundary integral formulation in [14], while a numerical implementation via integral equations is illustrated in [15]. As a matter of fact, the immediate utility of such models incorporates with one of the main fields of real-life applications nowadays, which is the Earth's subsurface electromagnetic probing for mineral exploration [16], identification of cavities [17] or other underground detections for UneXploded Ordinance [18, 19], and generally recovering buried obstacles [20], without excluding other useful physical applications interlacing with electromagnetic scattering by voluminous targets, illuminated either at low or at high frequencies. The idea developed here is much related to the full asymptotic expansions for general shaped permeable domains derived in [21], which are expressed in terms of the generalized polarization tensors and converge as the conductivity goes to zero.

In the investigation summarized herein, we inherit the diffusive scattering theory [1] and we cope with the problem of identifying a metallic body in an otherwise conductive medium, representing it as a general triaxial ellipsoid with arbitrary center, semiaxes lengths, and orientations, which embodies the complete anisotropy of the three-dimensional space. The object, excited by a time-harmonic magnetic dipole, operates at low frequency. Our devised modeling tools are based on a rigorous low-frequency analysis of the $3 \mathrm{D}$ vector electromagnetic fields (incident, scattered, and total ones) in positive integral powers of $(i k)^{n}$ for every order $n \geq 0, k$ denoting the complex-valued wave number of the exterior medium at the operation frequency. Therein, both their real and imaginary parts are of equivalent significance in the development of a reliable model. Then, our problem is transformed into a sequence of coupled boundary value problems for $n \geq 0$. Our analysis is confined to the most important terms of the expansions of the scattered fields, which are the static term for $n=0$ and the dynamic terms for $n=1,2,3$. The terms for $n \geq 4$ are considered very small, due to the low frequency in which the source operates and, consequently, they are neglected. Then, we mathematically formulate our analysis with respect to second-order Laplace's and Poisson's partial differential equations, completed with the appropriate perfectly reflecting boundary conditions, which comprised the cancellation of the normal magnetic and the tangential electric fields, while the Silver-Müller radiation conditions at infinity must automatically be satisfied as well. Hence, we face different well-posed boundary value problems for each case of $n=0,1,2,3$ as mentioned.

The important terms of the scattered fields are provided as infinite series expansions of ellipsoidal harmonic eigenfunctions $[2,4]$ in compact analytical fashion. In particular, the Rayleigh approximation static term for $n=0$ provides us only with a magnetic field of major importance, since it contributes mostly to the real part of the scattered magnetic field, while all the dynamic terms corresponding to $n=1$ are vanished as a result of the absence of incident fields. However, the most cumbersome case refers to the $n=2$ situation, where both the magnetic and the electric fields are present, occupying a significant percentage of the imaginary part of the scattered magnetic field and the entire one of the corresponding scattered electric field, respectively. Last but not least, the only surviving field at $n=3$ stands for a quite small correction to the real and imaginary part of the scattered magnetic field.

Although the majority of the solutions of physical applications in the ellipsoidal regime [2] uses only the few ellipsoidal harmonic functions that yield analytical closed-form expressions, in this project we manage to solve the aforementioned mathematical problem, introducing in a theoretical base, all the existing ellipsoidal harmonic eigenfunctions for any order and, therefore, of any degree. The efficiency of the model can be successfully demonstrated via the degeneration of the ellipsoidal shape and the reduction of the present results to the already known spheroidal [10] and spherical [7] analogous, since effective formulae of limiting procedures are given. On the other hand, the obtained analytical results are presented suchlike so as a numerical method could be employed furtherly as a continuation of this project. However, such method should be new and unique in the sense of using strong computational tools for evaluating ellipsoidal harmonics of higher orders until the accomplishment of the precise accuracy, where the potential series converge with the minimum of the needed effort. To imply that, we supplement the analytical section of this paper with a separate paragraph, whereas we provide all the necessary data values and the physical parameters for the scattering problem itself that simulates the Earth as the conductive medium and which contains the ellipsoidal anomalies. Then, any future numerical implementation must include plots that depict the 
variation of the measurable magnetic scattered field, as we move towards the surface.

\section{Physical and Mathematical Development}

We consider a solid ellipsoidal body with impenetrable surface $S$. The perfectly electrically conducting ellipsoid is embedded in a conductive, homogeneous, isotropic, and nonmagnetic medium of conductivity $\sigma$ and of permeability $\mu \cong \mu_{0}$ with $\mu_{0}$ being the permeability of free space, where, in terms of imaginary unit $i\left(i^{2}=-1\right)$, the complex-valued wave number is provided via

$$
k=\sqrt{i \omega \mu \sigma}=\sqrt{\frac{\omega \mu \sigma}{2}}(1+i)
$$

at a given low circular frequency $\omega$, while the dielectric permittivity $\varepsilon$ vanishes in such physical cases, since $\varepsilon \ll \sigma / \omega$. The external three-dimensional space $V\left(\mathbb{R}^{3}\right)$ is considered to be smooth and unbounded for our situation. Harmonic time dependence $\exp (-i \omega t)$ on all field quantities is implied; thus they are spatially coordinated by $\mathbf{r}=\sum_{j=1}^{3} x_{j} \widehat{\mathbf{x}}_{j}$ and expressed via the Cartesian basis $\widehat{\mathbf{x}}_{\kappa}, \kappa=1,2,3$ in Cartesian coordinates $\left(x_{1}, x_{2}, x_{3}\right)$, where this dependence will be omitted for writing convenience. The metallic ellipsoidal object is illuminated by a known magnetic dipole source

$$
\mathbf{m}=\sum_{j=1}^{3} m_{j} \widehat{\mathbf{x}}_{j},
$$

which is located at a precise position $\mathbf{r}_{0}$ and it is arbitrarily orientated, far away from the body. Then, the electromagnetic incident fields $\mathbf{H}^{i}$ and $\mathbf{E}^{i}$ are radiated by the magnetic dipole (2) and they are scattered by the solid ellipsoid, creating the scattered fields $\mathbf{H}^{s}$ and $\mathbf{E}^{\mathcal{s}}$, correspondingly. It holds that

$$
\begin{aligned}
\mathbf{H}^{t} & =\mathbf{H}^{i}+\mathbf{H}^{s}, \\
\mathbf{E}^{t} & =\mathbf{E}^{i}+\mathbf{E}^{\mathcal{s}},
\end{aligned}
$$

$$
\text { for } \mathbf{r} \in V\left(\mathbb{R}^{3}\right)-\left\{\mathbf{r}_{0}\right\}
$$

are the total magnetic and electric fields, given by the summation of the corresponding incident and scattered fields, where the singular point $\mathbf{r}_{0}$ has been excluded. Since the ellipsoidal metal body is nonpenetrable, there are no wave fields inside. By inheriting the low-frequency [1] diffusive theory, we construct the relative boundary value problems for the incident $(i)$, scattered $(s)$, and total $(t)$ electromagnetic fields through expansions in terms of powers of $(i k)$, such as

$$
\begin{aligned}
\mathbf{H}^{x} & =\sum_{n=0}^{\infty} \mathbf{H}_{n}^{x}(i k)^{n}, \\
\mathbf{E}^{x} & =\sum_{n=0}^{\infty} \mathbf{E}_{n}^{x}(i k)^{n}
\end{aligned}
$$

Thus, the well-known Maxwell's equations [1]

$$
\begin{aligned}
& \nabla \times \mathbf{E}^{x}=i \omega \mu \mathbf{H}^{x}, \\
& \nabla \times \mathbf{H}^{x}=(-i \omega \varepsilon+\sigma) \mathbf{E}^{x \stackrel{\varepsilon \ll \sigma / \omega}{\cong} \sigma \mathbf{E}^{x}}
\end{aligned}
$$

with $x=i, s, t$

are reduced into the low-frequency analogous

$$
\begin{aligned}
\sigma \nabla \times \mathbf{E}_{n}^{x}=-\mathbf{H}_{n-2}^{x} \quad \text { for } n \geq 2, \\
\nabla \times \mathbf{H}_{n}^{x}=\sigma \mathbf{E}_{n}^{x} \quad \text { for } n \geq 0 \\
\text { with } x=i, s, t,
\end{aligned}
$$

where in (5) and (6), the magnetic and electric fields are divergence-free for $\mathbf{r} \in V\left(\mathbb{R}^{3}\right)-\left\{\mathbf{r}_{0}\right\}$, yielding

$$
\begin{aligned}
& \nabla \cdot \mathbf{H}^{x}=\nabla \cdot \mathbf{E}^{x}=0, \\
& \nabla \cdot \mathbf{H}_{n}^{x}=\nabla \cdot \mathbf{E}_{n}^{x}=0
\end{aligned}
$$

for $n \geq 0$ with $x=i, s, t$.

The gradient operator $\nabla$ involved in relationships (5)-(7) operates at $\mathbf{r}$. But, it could also operate at $\mathbf{r}_{0}$; consequently for convenience we define as $\nabla=\sum_{j=1}^{3} \widehat{\mathbf{x}}_{j}\left(\partial / \partial x_{j}\right) \equiv \nabla_{\mathbf{r}}$ and similarly for the Laplacian operator $\Delta=\sum_{j=1}^{3}\left(\partial^{2} / \partial x_{j}^{2}\right) \equiv \Delta_{\mathbf{r}}$, unless it is said so.

For notational reasons we appoint as $\mathbf{R}=\mathbf{r}-\mathbf{r}_{0}$ and, hence, as $R=|\mathbf{R}|=\left|\mathbf{r}-\mathbf{r}_{0}\right|$; therefore, the electromagnetic incident fields generated by the magnetic dipole (2) assume the expressions

$$
\begin{aligned}
& \mathbf{H}^{i}=\frac{1}{4 \pi}\left[\left(k^{2}+\frac{i k}{R}-\frac{1}{R^{2}}\right) \mathbf{m}\right. \\
& \left.-\left(k^{2}+\frac{3 i k}{R}-\frac{3}{R^{2}}\right) \frac{\mathbf{R} \otimes \mathbf{R} \cdot \mathbf{m}}{R^{2}}\right] \frac{e^{i k R}}{R} \\
& \quad \text { for every } \mathbf{r} \in V\left(\mathbb{R}^{3}\right)-\left\{\mathbf{r}_{0}\right\}, \\
& \mathbf{E}^{i}=\left[\frac{\omega \mu k}{4 \pi}\left(1+\frac{i}{k R}\right) \frac{\mathbf{m} \times \mathbf{R}}{R}\right] \frac{e^{i k R}}{R} \\
& \text { for every } \mathbf{r} \in V\left(\mathbb{R}^{3}\right)-\left\{\mathbf{r}_{0}\right\},
\end{aligned}
$$

where the symbol " $\otimes$ " denotes juxtaposition between two vectors. Extended algebraic calculations on the incident fields (8), based on the Taylor's expansion of the exponential functions $e^{i k R}$ and on definition (1), yield low-frequency relations as powers of $(i k)$ for the incident fields. Then, the static term for $n=0$ and the dynamic terms for $n=1,2,3$, which are sufficient enough to describe the fields, since they live in the low-frequency regime, enjoy the relationships

$$
\mathbf{H}^{i}=\left[\mathbf{H}_{0}^{i}+\mathbf{H}_{2}^{i}(i k)^{2}+\mathbf{H}_{3}^{i}(i k)^{3}\right]+\bigcirc\left((i k)^{4}\right)
$$

$$
\text { for every } \mathbf{r} \in V\left(\mathbb{R}^{3}\right)-\left\{\mathbf{r}_{0}\right\} \text {, }
$$




$$
\begin{aligned}
\mathbf{E}^{i}=\left[\mathbf{E}_{2}^{i}(i k)^{2}\right]+ & O\left((i k)^{4}\right) \\
& \text { for every } \mathbf{r} \in V\left(\mathbb{R}^{3}\right)-\left\{\mathbf{r}_{0}\right\},
\end{aligned}
$$

whereas, in view of the unit dyadic $\widetilde{\mathbf{I}}=\sum_{j=1}^{3} \widehat{\mathbf{x}}_{j} \otimes \widehat{\mathbf{x}}_{j}$, we obtain

$$
\begin{aligned}
\mathbf{H}_{0}^{i} & =\frac{\mathbf{m}}{4 \pi} \cdot\left(\frac{3 \mathbf{R} \otimes \mathbf{R}}{R^{2}}-\widetilde{\mathbf{I}}\right) \frac{1}{R^{3}}=\frac{\mathbf{m}}{4 \pi} \cdot\left(\nabla \otimes \nabla \frac{1}{R}\right), \\
\mathbf{H}_{2}^{i} & =-\frac{\mathbf{m}}{4 \pi} \cdot\left(\frac{\mathbf{R} \otimes \mathbf{R}}{R^{2}}+\widetilde{\mathbf{I}}\right) \frac{1}{2 R} \\
& =\frac{\mathbf{m}}{4 \pi} \cdot \frac{1}{2}\left(\nabla \frac{1}{R} \otimes \mathbf{R}-\frac{\widetilde{\mathbf{I}}}{R}\right), \\
\mathbf{H}_{3}^{i} & =\frac{\mathbf{m}}{4 \pi} \cdot\left(-\frac{2}{3} \widetilde{\mathbf{I}}\right)
\end{aligned}
$$

for the incident magnetic fields, while

$$
\mathbf{E}_{2}^{i}=-\frac{\mathbf{m}}{4 \pi \sigma} \times \frac{\mathbf{R}}{R^{3}}=\frac{\mathbf{m}}{4 \pi \sigma} \times \nabla \frac{1}{R}
$$

for the incident electric field. The derivation of the second equivalent, but easy-to-handle, differential forms on the right-hand side of the nontrivial incident fields (11)-(14), defined for $\mathbf{r} \in V\left(\mathbb{R}^{3}\right)-\left\{\mathbf{r}_{0}\right\}$, is straightforward and it is based on the fact that

$$
\begin{aligned}
& \nabla_{\mathbf{r}} \frac{1}{R} \equiv \nabla \frac{1}{R}=-\frac{\mathbf{R}}{R^{3}}, \\
& \nabla_{\mathbf{r}} \frac{1}{R} \equiv \nabla \frac{1}{R}=-\nabla_{\mathbf{r}_{0}} \frac{1}{R}
\end{aligned}
$$

$$
\text { for every } \mathbf{r} \in V\left(\mathbb{R}^{3}\right)-\left\{\mathbf{r}_{0}\right\} \text {, }
$$

given $\nabla \otimes \mathbf{r}=\widetilde{\mathbf{I}}$, along with the use of trivial differential identities. It is clear that the magnetic terms of any order $n \geq 0$ vary like $1 / R^{3-n}$, while the electric ones vary like $1 / R^{4-n}$ as $R$ goes to infinity. An immediate observation reveals that for the incident magnetic field the dynamic term for $n=1$ is not present, while for the incident electric field the only term that survives is the dynamic term for $n=2$, reflecting exactly the same physical and mathematical attribute to the scattered fields.

Hence, for the low-frequency orders of interest $n=0,2,3$ (note that for $n=1$ we have no fields at all), the scattered magnetic field

$$
\begin{aligned}
\mathbf{H}^{s}=\left[\mathbf{H}_{0}^{s}+\mathbf{H}_{2}^{s}(i k)^{2}+\mathbf{H}_{3}^{s}(i k)^{3}\right]+O\left((i k)^{4}\right) \\
\\
\quad \text { for every } \mathbf{r} \in V\left(\mathbb{R}^{3}\right)-\left\{\mathbf{r}_{0}\right\}
\end{aligned}
$$

and the scattered electric field,

$$
\begin{aligned}
\mathbf{E}^{s}=\left[\mathbf{E}_{2}^{s}(i k)^{2}\right]+ & O\left((i k)^{4}\right) \\
& \text { for every } \mathbf{r} \in V\left(\mathbb{R}^{3}\right)-\left\{\mathbf{r}_{0}\right\},
\end{aligned}
$$

inherit similar forms to those of the incident fields (9) and (10), respectively, where the fields $\mathbf{H}_{0}^{s}, \mathbf{H}_{2}^{s}, \mathbf{H}_{3}^{s}$, and $\mathbf{E}_{2}^{s}$ are to be evaluated. In the aim of separating real and imaginary parts to the scattered fields, we substitute the wave number of the surrounding medium (1) into relations (16) and (17), whereas after some trivial analysis we are led to

$$
\begin{aligned}
\mathbf{H}^{s}= & {\left[\mathbf{H}_{0}^{s}+(\omega \mu \sigma) \sqrt{\frac{\omega \mu \sigma}{2}} \mathbf{H}_{3}^{s}\right] } \\
+(\omega \mu \sigma)\left[\sqrt{\frac{\omega \mu \sigma}{2}} \mathbf{H}_{3}^{s}-\mathbf{H}_{2}^{s}\right] i+\bigcirc\left((i k)^{4}\right) & \text { for every } \mathbf{r} \in V\left(\mathbb{R}^{3}\right)-\left\{\mathbf{r}_{0}\right\}, \\
\mathbf{E}^{s}=\left[-(\omega \mu \sigma) \mathbf{E}_{2}^{s}\right] i+\bigcirc\left((i k)^{4}\right) & \text { for every } \mathbf{r} \in V\left(\mathbb{R}^{3}\right)-\left\{\mathbf{r}_{0}\right\},
\end{aligned}
$$

respectively. The electric field (19) is purely imaginary-valued, needing only $\mathbf{E}_{2}^{\mathcal{S}}$, while the magnetic field (18) is complexvalued, noticing that the magnetic field at order $n=2$ $\left(\mathbf{H}_{2}^{s}\right)$ is adequate for the imaginary part, while the zero-order static term $\mathbf{H}_{0}^{s}$ yields a very good approximation for the real part. The contribution of $\mathbf{H}_{3}^{s}$, as the outcome of the constant field (13), stands for a very small correction to both real and imaginary parts of the scattered magnetic field (18), while the first-order $(n=1)$ field $\mathbf{H}_{1}^{s}$ is absent, in absence of incident fields at that order.

In that sense, straightforward calculations on Maxwell's equations (6) for $x=s$ and elaborate use of identity $\nabla \times \nabla \times$ $\mathbf{f}=\nabla(\nabla \cdot \mathbf{f})-\Delta \mathbf{f}$ with $\mathbf{f}$ being any vector result in the mixed Maxwell-type boundary value problems

$$
\begin{aligned}
& \Delta \mathbf{H}_{0}^{s}=\mathbf{0} \Longrightarrow \mathbf{H}_{0}^{s}=\nabla \Phi_{0}^{s}, \\
& \text { since } \nabla \cdot \mathbf{H}_{0}^{s}=0, \nabla \times \mathbf{H}_{0}^{s}=\mathbf{0} \text {, } \\
& \Delta \mathbf{H}_{2}^{s}=\mathbf{H}_{0}^{s} \Longrightarrow \mathbf{H}_{2}^{s}=\Phi_{2}^{s}+\frac{1}{2}\left(\mathbf{r} \Phi_{0}^{s}\right), \\
& \sigma \mathbf{E}_{2}^{s}=\nabla \times \mathbf{H}_{2}^{s} \text {, }
\end{aligned}
$$$$
\text { since } \nabla \cdot \mathbf{H}_{2}^{s}=\nabla \cdot \mathbf{E}_{2}^{s}=0 \text {, }
$$$$
\Delta \mathbf{H}_{3}^{s}=\mathbf{0} \Longrightarrow \mathbf{H}_{3}^{s}=\nabla \Phi_{3}^{s},
$$$$
\text { since } \nabla \cdot \mathbf{H}_{3}^{s}=0, \nabla \times \mathbf{H}_{3}^{s}=\mathbf{0} \text {, }
$$

which are written in terms of the harmonic potentials $\Phi_{0}^{s}$, $\Phi_{2}^{s}$ and $\Phi_{3}^{s}$ that satisfy the following classical Laplace's partial differential equations:

$$
\begin{aligned}
\Delta \Phi_{0}^{s} & =\Delta \Phi_{3}^{s}=0, \\
\Delta \Phi_{2}^{s} & =\mathbf{0} .
\end{aligned}
$$

The scattered fields $\mathbf{H}_{0}^{s}, \mathbf{H}_{2}^{s}, \mathbf{E}_{2}^{s}$, and $\mathbf{H}_{3}^{s}$ must be calculated in the prescribed scattering domain $V\left(\mathbb{R}^{3}\right)-\left\{\mathbf{r}_{0}\right\}$, while $\mathbf{H}_{1}^{s}=\mathbf{E}_{0}^{s}=\mathbf{E}_{1}^{s}=\mathbf{E}_{3}^{s}=\mathbf{0}$ as direct consequence of the incident fields (9) and (10) and Maxwell's equations (6). It is worth mentioning that for $n=0,3$ standard Laplace's equations must be solved for the $\mathbf{H}_{0}^{s}$ and $\mathbf{H}_{3}^{s}$ fields, while 
the inhomogeneous vector Laplace equation (21), coupled with the solution of (20), is Poisson's partial differential equation. Provided that the zero-order scattered field $\mathbf{H}_{0}^{s}$ is obtained, the second-order scattered field $\mathbf{H}_{2}^{s}$ can be written as a general vector harmonic function $\Phi_{2}^{s}$ plus a particular solution $(1 / 2)\left(\mathbf{r} \Phi_{0}^{s}\right)$, where it is ensured straightforwardly that

$$
\Delta\left(\frac{1}{2} \mathbf{r} \Phi_{0}^{s}\right)=(\nabla \otimes \mathbf{r}) \cdot \nabla \Phi_{0}^{s}=\widetilde{\mathbf{I}} \cdot \nabla \Phi_{0}^{s}=\nabla \Phi_{0}^{s} \equiv \mathbf{H}_{0}^{s},
$$

as a consequence of (20), as well as the harmonic character of both the position vector $\mathbf{r}$ and the potential $\Phi_{0}^{s}$. Finally, the scattered electric field $\mathbf{E}_{2}^{s}$ for $n=2$, it is given by the rotational action of the gradient operator on the corresponding magnetic field via (21).

The set of low-frequency problems (20)-(23) is accompanied by the proper perfectly electrically conducting boundary conditions on the surface $S$ of the ellipsoidal target. They concern the total fields (3) at each preferable order $n=0,2,3$, where, by definition of the outward unit normal vector $\widehat{\mathbf{n}}$, the normal component of the total magnetic field and the tangential component of the total electric field are canceled; that is,

$$
\begin{aligned}
& \widehat{\mathbf{n}} \cdot \mathbf{H}^{t}=0, \\
& \widehat{\mathbf{n}} \times \mathbf{E}^{t}=\mathbf{0}
\end{aligned}
$$

for every $\mathbf{r} \in S$,

respectively. Hence, combining (3) and (4) with (25), we readily obtain

$$
\begin{array}{ll}
\widehat{\mathbf{n}} \cdot\left(\mathbf{H}_{n}^{i}+\mathbf{H}_{n}^{s}\right)=0 & \text { with } n=0,2,3, \\
\widehat{\mathbf{n}} \times\left(\mathbf{E}_{2}^{i}+\mathbf{E}_{2}^{s}\right)=\mathbf{0} & \text { for every } \mathbf{r} \in S .
\end{array}
$$

Additionally, the Silver-Müller radiation conditions at infinity for the scattered fields

$$
\begin{aligned}
\lim _{|\mathbf{r}| \rightarrow+\infty}\left[\mathbf{r} \times \nabla \times\left(\begin{array}{c}
\mathbf{H}^{s} \\
\mathbf{E}^{s}
\end{array}\right)+\right. & \left.i k|\mathbf{r}|\left(\begin{array}{c}
\mathbf{H}^{s} \\
\mathbf{E}^{s}
\end{array}\right)\right]=\mathbf{0} \\
& \text { for every } \mathbf{r} \in V\left(\mathbb{R}^{3}\right)-\left\{\mathbf{r}_{0}\right\}
\end{aligned}
$$

must automatically be satisfied, which, in view of (4), are written as

$$
\lim _{|\mathbf{r}| \rightarrow+\infty}\left[\mathbf{r} \times \nabla \times\left(\begin{array}{c}
\mathbf{H}_{n}^{s} \\
\mathbf{E}_{n}^{s}
\end{array}\right)+|\mathbf{r}|\left(\begin{array}{c}
\mathbf{H}_{n-1}^{s} \\
\mathbf{E}_{n-1}^{s}
\end{array}\right)\right]=\mathbf{0}
$$

with $n=2,3$ for every $\mathbf{r} \in V\left(\mathbb{R}^{3}\right)-\left\{\mathbf{r}_{0}\right\}$,

since for $n=1$ there are no fields, while for $n=0$ it is verified from (20) that $\lim _{|\mathbf{r}| \rightarrow+\infty}\left(\mathbf{r} \times \nabla \times \mathbf{H}_{0}^{s}\right)=\mathbf{0}$, where $\mathbf{E}_{0}^{s}=\mathbf{0}$. Solutions with exterior behavior, as in our case, satisfy (28) automatically, resulting from the appropriate elaboration of the corresponding eigenfunctions.

Recapitulating, we are ready to apply the particular ellipsoidal geometry [2-4] in a proper manner to solve the aforementioned boundary value problems to recover the electromagnetic fields. Those are the static magnetic one for $n=0$, reduced to a potential problem with Neumann-type boundary condition, the electric and magnetic one for $n=2$, where the problem is far more complicated due to coupling to the static term, where the scattered electric field for $n=2$ is given through the second part of relationship (21), and the one for $n=3$, which comprise again a potential problem with Neumann boundary condition for the corresponding magnetic field.

\section{Ellipsoidal Geometry and Harmonic Analysis}

In this section we invoke principal information concerning the geometry and the harmonic analysis of the ellipsoidal coordinate system, where more analytical information can be found in [2]. The basic triaxial ellipsoid, which embodies the complete anisotropy of the three-dimensional space, is defined by

$$
\sum_{j=1}^{3} \frac{x_{j}^{2}}{\alpha_{j}^{2}}=1
$$

where $0<\alpha_{3}<\alpha_{2}<\alpha_{1}<+\infty$ are its semiaxes. The three positive numbers

$$
\begin{aligned}
& h_{1}=\sqrt{\alpha_{2}^{2}-\alpha_{3}^{2}}, \\
& h_{2}=\sqrt{\alpha_{1}^{2}-\alpha_{3}^{2}}, \\
& h_{3}=\sqrt{\alpha_{1}^{2}-\alpha_{2}^{2}},
\end{aligned}
$$

$$
\text { where } h_{3}^{2}=h_{2}^{2}-h_{1}^{2}
$$

denote the semifocal distances of the ellipsoidal system, whose coordinates $(\rho, \mu, \nu)$ are connected to the Cartesian ones $\left(x_{1}, x_{2}, x_{3}\right)$ via the expressions

$$
\begin{aligned}
& x_{\kappa}=\frac{h_{\kappa}}{h_{1} h_{2} h_{3}} \\
& \cdot \sqrt{\left|\rho^{2}-\alpha_{1}^{2}+\alpha_{\kappa}^{2}\right|} \sqrt{\left|\mu^{2}-\alpha_{1}^{2}+\alpha_{\kappa}^{2}\right|} \sqrt{\left|\nu^{2}-\alpha_{1}^{2}+\alpha_{\kappa}^{2}\right|} \\
& \text { for } \kappa=1,2,3,
\end{aligned}
$$

within the prescribed intervals $\rho \in\left[h_{2},+\infty\right), \mu \in\left[h_{3}, h_{2}\right]$, and $v \in\left[0, h_{3}\right]$, such as the sequences of the inequalities $0 \leq v^{2} \leq h_{3}^{2} \leq \mu^{2} \leq h_{2}^{2} \leq \rho^{2}<+\infty$ holding true. The three families of second-degree surfaces, which are shown in Figure 1, share the same set of foci at the points $\pm h_{1}, \pm h_{2}$ and $\pm h_{3}$.

In view of the position vector $\mathbf{r}$ with measure $\|\mathbf{r}\|=$ $\sqrt{\sum_{j=1}^{3} x_{j}^{2}}=\sqrt{\rho^{2}+\mu^{2}+v^{2}-\left(h_{2}^{2}+h_{3}^{2}\right)}$, the radial-like variable $h_{2} \leq \rho<+\infty$ specifies the ellipsoid

$$
\sum_{j=1}^{3} \frac{x_{j}^{2}}{\rho^{2}-\alpha_{1}^{2}+\alpha_{j}^{2}}=\mathbf{r} \cdot \sum_{j=1}^{3} \frac{\widehat{\mathbf{x}}_{j} \otimes \widehat{\mathbf{x}}_{j}}{\rho^{2}-\alpha_{1}^{2}+\alpha_{j}^{2}} \cdot \mathbf{r}=1,
$$




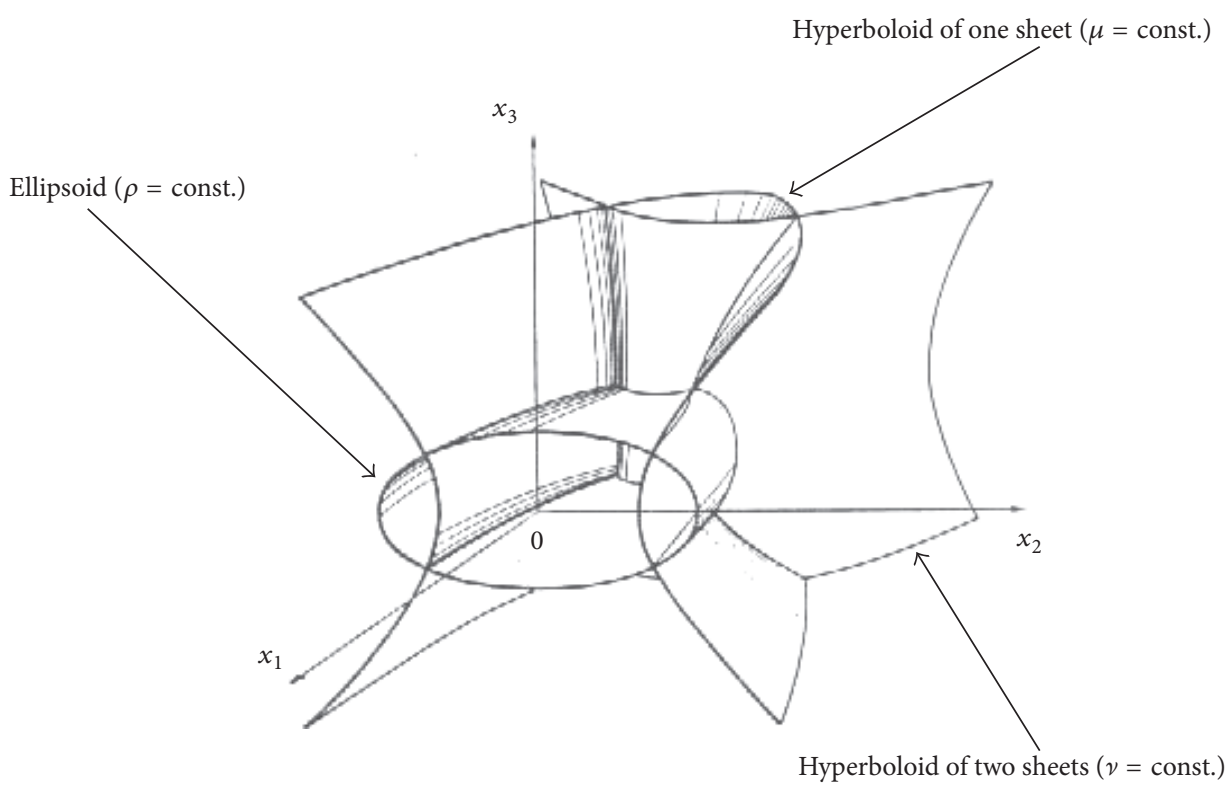

FIGURE 1: Ellipsoidal geometry and coordinate surfaces.

and the variable $h_{3} \leq \mu \leq h_{2}$ denotes the hyperboloid of one sheet

$$
\sum_{j=1}^{3} \frac{x_{j}^{2}}{\mu^{2}-\alpha_{1}^{2}+\alpha_{j}^{2}}=1,
$$

while the variable $0 \leq v \leq h_{3}$ gives the hyperboloid of two sheets:

$$
\sum_{j=1}^{3} \frac{x_{j}^{2}}{v^{2}-\alpha_{1}^{2}+\alpha_{j}^{2}}=1
$$

In terms of the metric coefficients of the ellipsoidal coordinate system

$$
\begin{aligned}
& h_{\rho}=\frac{\sqrt{\rho^{2}-\mu^{2}} \sqrt{\rho^{2}-v^{2}}}{\sqrt{\rho^{2}-h_{3}^{2}} \sqrt{\rho^{2}-h_{2}^{2}}}, \\
& h_{\mu}=\frac{\sqrt{\rho^{2}-\mu^{2}} \sqrt{\mu^{2}-v^{2}}}{\sqrt{\mu^{2}-h_{3}^{2}} \sqrt{h_{2}^{2}-\mu^{2}}}, \\
& h_{\nu}=\frac{\sqrt{\rho^{2}-v^{2}} \sqrt{\mu^{2}-v^{2}}}{\sqrt{h_{3}^{2}-v^{2}} \sqrt{h_{2}^{2}-v^{2}}},
\end{aligned}
$$

as well as the Jacobian determinant $J=h_{\rho} h_{\mu} h_{v}$ for every $\rho \in\left[h_{2},+\infty\right), \mu \in\left[h_{3}, h_{2}\right]$, and $\nu \in\left[0, h_{3}\right]$, the differential operators

$$
\nabla=\frac{\widehat{\boldsymbol{\rho}}}{h_{\rho}} \frac{\partial}{\partial \rho}+\frac{\widehat{\boldsymbol{\mu}}}{h_{\mu}} \frac{\partial}{\partial \mu}+\frac{\widehat{\boldsymbol{\nu}}}{h_{\nu}} \frac{\partial}{\partial \nu},
$$

$\Delta$

$$
=\frac{1}{J}\left\{\frac{\partial}{\partial \rho}\left[\frac{J}{h_{\rho}^{2}} \frac{\partial}{\partial \rho}\right]+\frac{\partial}{\partial \mu}\left[\frac{J}{h_{\mu}^{2}} \frac{\partial}{\partial \mu}\right]+\frac{\partial}{\partial \nu}\left[\frac{J}{h_{\nu}^{2}} \frac{\partial}{\partial \nu}\right]\right\}
$$

stand for the gradient and Laplace's operators in ellipsoidal geometry, respectively, written via the orthonormal coordinate vectors of the system

$$
\begin{aligned}
& \widehat{\boldsymbol{\rho}}=\frac{\rho}{h_{\rho}} \sum_{j=1}^{3} \frac{x_{j} \widehat{\mathbf{x}}_{j}}{\rho^{2}-\alpha_{1}^{2}+\alpha_{j}^{2}}, \\
& \widehat{\boldsymbol{\mu}}=\frac{\mu}{h_{\mu}} \sum_{j=1}^{3} \frac{x_{j} \widehat{\mathbf{x}}_{j}}{\mu^{2}-\alpha_{1}^{2}+\alpha_{j}^{2}}, \\
& \widehat{\boldsymbol{\nu}}=\frac{\nu}{h_{\nu}} \sum_{j=1}^{3} \frac{x_{j} \widehat{\mathbf{x}}_{j}}{v^{2}-\alpha_{1}^{2}+\alpha_{j}^{2}} .
\end{aligned}
$$

The outward unit normal vector on the surface of any ellipsoid $\rho=$ const., given through

$$
\widehat{\mathbf{n}}=\frac{\rho}{h_{\rho}} \sum_{j=1}^{3} \frac{\widehat{\mathbf{x}}_{j} \otimes \widehat{\mathbf{x}}_{j}}{\rho^{2}-\alpha_{1}^{2}+\alpha_{j}^{2}} \cdot \mathbf{r} \equiv \widehat{\boldsymbol{\rho}}
$$

for every $\rho \in\left[h_{2},+\infty\right), \mu \in\left[h_{3}, h_{2}\right], v \in\left[0, h_{3}\right]$,

coincides with the unit normal vector $\widehat{\rho}$. On the other hand, the unit dyadic in ellipsoidal coordinates yields

$$
\widetilde{\mathbf{I}}=\widehat{\boldsymbol{\rho}} \otimes \widehat{\boldsymbol{\rho}}+\widehat{\boldsymbol{\mu}} \otimes \widehat{\boldsymbol{\mu}}+\widehat{\boldsymbol{\nu}} \otimes \widehat{\nu},
$$


where we provide the useful relationship

$$
\begin{aligned}
\nabla \otimes \widehat{\boldsymbol{\rho}}= & \frac{\rho}{h_{\rho}}\left[\frac{\widehat{\boldsymbol{\mu}} \otimes \widehat{\boldsymbol{\mu}}}{\rho^{2}-\mu^{2}}+\frac{\widehat{\boldsymbol{\nu}} \otimes \widehat{\boldsymbol{\nu}}}{\rho^{2}-v^{2}}\right]+\frac{\mu}{h_{\mu}} \frac{\widehat{\boldsymbol{\rho}} \otimes \widehat{\boldsymbol{\mu}}}{\rho^{2}-\mu^{2}} \\
& +\frac{\nu}{h_{\nu}} \frac{\widehat{\boldsymbol{\rho}} \otimes \widehat{\boldsymbol{\nu}}}{\rho^{2}-v^{2}},
\end{aligned}
$$

by which one can recover the products $\nabla \cdot \widehat{\boldsymbol{\rho}}$ and $\nabla \times \widehat{\boldsymbol{\rho}}$ in an easy manner.

In order to represent harmonic potentials that belong to the kernel space of Laplace's operator (37), we need to construct the appropriate harmonic eigenfunctions, which will provide us with the corresponding eigensolutions in spectral form. This procedure leads to the Lamé equation:

$$
\begin{aligned}
& \left(x^{2}-h_{3}^{2}\right)\left(x^{2}-h_{2}^{2}\right) E^{\prime \prime}(x)+x\left(2 x^{2}-h_{3}^{2}-h_{2}^{2}\right) E^{\prime}(x) \\
& +\left(A x^{2}+B\right) E(x)=0,
\end{aligned}
$$

where the prime denotes derivation with respect to the argument and $A, B \in \mathbb{R}$ are constants, while we denote $x \equiv$ $\rho, \mu, \nu$ for each one of the factors $E(\rho), E(\mu)$, and $E(\nu)$ within the corresponding intervals $\rho \in\left[h_{2},+\infty\right), \mu \in\left[h_{3}, h_{2}\right]$, and $v \in\left[0, h_{3}\right]$. For each $\ell=0,1,2, \ldots$, which corresponds to the degree of the Lamé equation and for each $m=1,2, \ldots, 2 \ell+1$, which stands for its order, (42) has two linearly independent solutions. The first one, $E_{\ell}^{m}$, is regular at the origin and it is known as the Lamé function of the first kind, yielding to interior solutions, while the second one $F_{\ell}^{m}$ is regular at infinity and gives the Lamé function of the second kind, corresponding to exterior solutions. In particular, for $\ell \geq 0$ and $m=1,2, \ldots, 2 \ell+1$, the interior function $E_{\ell}^{m}(\rho)$ is related to the exterior one $F_{\ell}^{m}(\rho)$ via the expression

$$
\begin{aligned}
& F_{\ell}^{m}(\rho)=(2 \ell+1) E_{\ell}^{m}(\rho) I_{\ell}^{m}(\rho) \\
& \quad \text { for every } \rho \in\left[h_{2},+\infty\right)
\end{aligned}
$$

and by definition of the elliptic integrals and their derivatives with respect to $\rho \in\left[h_{2},+\infty\right)$,

$$
\begin{aligned}
I_{\ell}^{m}(\rho) & =\int_{\rho}^{+\infty} \frac{d u}{\left[E_{\ell}^{m}(u)\right]^{2} \sqrt{u^{2}-h_{2}^{2}} \sqrt{u^{2}-h_{3}^{2}}} \Longrightarrow \\
\frac{d I_{\ell}^{m}(\rho)}{d \rho} & =-\frac{1}{\left[E_{\ell}^{m}(\rho)\right]^{2} \sqrt{\rho^{2}-h_{3}^{2}} \sqrt{\rho^{2}-h_{2}^{2}}},
\end{aligned}
$$

respectively. In terms of the Lamé functions of the first and of the second kind for any degree of preference $\ell=0,1,2, \ldots$ and order $m=1,2, \ldots, 2 \ell+1$, the Lamé products

$$
\begin{aligned}
& \mathbb{E}_{\ell}^{m}=E_{\ell}^{m}(\rho) S_{\ell}^{m}(\mu, \nu) \\
& \quad \text { for every } \rho \in\left[h_{2},+\infty\right), \mu \in\left[h_{3}, h_{2}\right], \nu \in\left[0, h_{3}\right]
\end{aligned}
$$

define the interior solid ellipsoidal harmonic eigenfunctions, while the products

$$
\begin{aligned}
& \mathbb{F}_{\ell}^{m}=F_{\ell}^{m}(\rho) S_{\ell}^{m}(\mu, \nu)=(2 \ell+1) I_{\ell}^{m}(\rho) \mathbb{E}_{\ell}^{m} \\
& \quad \text { for every } \rho \in\left[h_{2},+\infty\right), \mu \in\left[h_{3}, h_{2}\right], \nu \in\left[0, h_{3}\right],
\end{aligned}
$$

in view of (42), comprise the exterior solid ellipsoidal harmonics. The complete orthogonal set

$$
\begin{aligned}
& S_{\ell}^{m}(\mu, \nu)=E_{\ell}^{m}(\mu) E_{\ell}^{m}(\nu) \\
& \quad \text { for every } \rho \in\left[h_{2},+\infty\right), \mu \in\left[h_{3}, h_{2}\right], \nu \in\left[0, h_{3}\right],
\end{aligned}
$$

form the surface ellipsoidal harmonics on the surface of any prescribed ellipsoid $\rho=\rho_{s}$, which, with respect to the weighting function factor $\left[\left(\rho_{s}^{2}-\mu^{2}\right)\left(\rho_{s}^{2}-v^{2}\right)\right]^{-1 / 2}$ for every $\mu \in\left[h_{3}, h_{2}\right]$ and $\nu \in\left[0, h_{3}\right]$, satisfy the orthogonality relation

$$
\begin{gathered}
\iint_{\rho=\rho_{s}} S_{\ell}^{m}(\mu, \nu) S_{\ell^{\prime}}^{m^{\prime}}(\mu, \nu) \frac{d s(\mu, \nu)}{\sqrt{\left(\rho_{s}^{2}-\mu^{2}\right)\left(\rho_{s}^{2}-v^{2}\right)}} \\
\quad=\gamma_{\ell}^{m} \delta_{\ell \ell^{\prime}} \delta_{m m^{\prime}} \quad \text { with } d s(\mu, \nu)=h_{\mu} h_{\nu} d \mu d \nu
\end{gathered}
$$

for $\ell \geq 0$ and $m=1,2, \ldots, 2 \ell+1$, where $\delta$-symbol is the kronecker delta and the ellipsoidal normalization constants read as

$$
\begin{aligned}
\gamma_{\ell}^{m}=\iint_{\rho=\rho_{s}}\left[S_{\ell}^{m}(\mu, \nu)\right]^{2} \frac{d s(\mu, \nu)}{\sqrt{\left(\rho_{s}^{2}-\mu^{2}\right)\left(\rho_{s}^{2}-\nu^{2}\right)}} \\
\text { for } \ell \geq 0, m=1,2, \ldots, 2 \ell+1 .
\end{aligned}
$$

Therein, any scalar harmonic function $f$, which could be vector as well, solves Laplace's equation $\Delta f=0$ and assumes the expansion

$$
\begin{aligned}
& f=\sum_{\ell=0}^{\infty} \sum_{m=1}^{2 \ell+1}\left(A_{\ell}^{m} \mathbb{E}_{\ell}^{m}+B_{\ell}^{m} \mathbb{F}_{\ell}^{m}\right) \\
& \text { for every } \rho \in\left[h_{2},+\infty\right), \mu \in\left[h_{3}, h_{2}\right], \nu \in\left[0, h_{3}\right],
\end{aligned}
$$

where $A_{\ell}^{m}$ and $B_{\ell}^{m}$ for $\ell \geq 0$ and $m=1,2, \ldots, 2 \ell+1$ are unknown constant coefficients, while every smooth and welldefined function $g(\mu, v)$ is expanded on the surface of the ellipsoid $\rho=\rho_{s}$ in terms of the ellipsoidal orthonormal basis according to

$$
\begin{aligned}
g(\mu, \nu)=\sum_{\ell=0}^{\infty} \sum_{m=1}^{2 \ell+1} C_{\ell}^{m} S_{\ell}^{m}(\mu, \nu) & \\
& \quad \text { for every } \mu \in\left[h_{3}, h_{2}\right], \nu \in\left[0, h_{3}\right],
\end{aligned}
$$

where, by virtue of (48), the constant coefficients $C_{\ell}^{m}$ admit

$$
\begin{array}{r}
C_{\ell}^{m} \\
=\frac{1}{\gamma_{\ell}^{m}} \iint_{\rho=\rho_{s}} g(\mu, \nu) S_{\ell}^{m}(\mu, \nu) \frac{d s(\mu, \nu)}{\sqrt{\left(\rho_{s}^{2}-\mu^{2}\right)\left(\rho_{s}^{2}-v^{2}\right)}} \\
\quad \text { for } \ell \geq 0, m=1,2, \ldots, 2 \ell+1 .
\end{array}
$$

Finally, in order to collect the basic tools for solving boundary value problems in fundamental domains with ellipsoidal boundaries, we introduce Heine's expansion formulae for any 
singular point $\mathbf{r}_{0}$, which express the fundamental solution of the Laplacian in terms of ellipsoidal harmonics as

$$
\begin{aligned}
& \frac{1}{R} \equiv \frac{1}{\left|\mathbf{r}-\mathbf{r}_{0}\right|} \\
& =\sum_{\ell=0}^{\infty} \sum_{m=1}^{2 \ell+1} \frac{4 \pi}{(2 \ell+1) \gamma_{\ell}^{m}} \begin{cases}\mathbb{F}_{\ell}^{m}\left(\mathbf{r}_{0}\right) \mathbb{E}_{\ell}^{m}, & \rho<\rho_{0} \\
\mathbb{E}_{\ell}^{m}\left(\mathbf{r}_{0}\right) \mathbb{F}_{\ell}^{m}, & \rho>\rho_{0}\end{cases} \\
& \text { with } \mathbf{r}_{0}=\left(\rho_{0}, \mu_{0}, v_{0}\right)
\end{aligned}
$$

for every $\rho \in\left[h_{2},+\infty\right), \mu \in\left[h_{3}, h_{2}\right]$ and $\nu \in\left[0, h_{3}\right]$.

The strict inequalities $0<\alpha_{3}<\alpha_{2}<\alpha_{1}<+\infty$ form the basic reason why the triaxial ellipsoid reflects the general anisotropy of the three-dimensional space. As it is well-known, the reduction of general results from the ellipsoidal to the spheroidal or to the spherical geometry is not straightforward, since certain indeterminacies appear during the limiting process. This is due to the fact that the spherical system springs from a zero-dimensional manifold, that is, the center, while the ellipsoidal system springs from a two-dimensional manifold, that is, the focal ellipse. The equality of any of the two axes of an ellipsoid degenerates it to a spheroid, whose axial symmetry coincides with the third axis. More specific, a prolate spheroid is obtained whenever $0<\alpha_{3}=\alpha_{2}<\alpha_{1}<+\infty$ (with the semifocal distances taken as $h_{1}=0$ and $h_{2}=h_{3}=c>0$ ), while the case of an oblate spheroidal shape corresponds to $0<\alpha_{3}<\alpha_{2}=$ $\alpha_{1}<+\infty$ (with the semifocal distances taken as $h_{3}=0$ and $h_{1}=h_{2}=\bar{c}>0$ ). The axis of symmetry is the $x_{1}$-axis for the prolate spheroid and the $x_{3}$-axis for the oblate spheroid. The asymptotic case of the needle can be reached by a prolate spheroid where $0<\alpha_{3}=\alpha_{2} \ll \alpha_{1}<+\infty$, while in the case where $0<\alpha_{3} \ll \alpha_{2}=\alpha_{1}<+\infty$ the oblate spheroid takes the shape of a circular disk. The simple transformation $c \rightarrow-i \bar{c}$ allows the transition from the prolate to the oblate spheroid, while the replacement $\bar{c} \rightarrow$ ic secures the converse. On the other hand, the sphere situation corresponds to $\alpha_{1}=\alpha_{2}=$ $\alpha_{3}=\alpha$, where $\alpha>0$ is the radius, while in this case $h_{\kappa}=0$ for $\kappa=1,2,3$, which means that all the semifocal distances of the ellipsoid coincide at the origin.

In terms of the variables the above limiting process becomes slightly more complicated. Hence, we introduce the prolate spheroidal coordinates $(\tau \equiv \cosh \eta, \zeta \equiv \cos \theta, \varphi)$ with $\tau \in[1,+\infty), \zeta \in[-1,1]$ and $\varphi \in[0,2 \pi)$ (note that the oblate geometry $(\lambda \equiv \sinh \eta, \zeta \equiv \cos \theta, \varphi)$ with $\lambda \in[0,+\infty)$ is recovered via the transformation $\tau \rightarrow i \lambda$, while the inverse one $\lambda \rightarrow-i \tau$ secures the opposite), as well as the spherical coordinates $(r, \zeta \equiv \cos \theta, \varphi)$ with $r \in[0,+\infty), \zeta \in[-1,1]$ and $\varphi \in[0,2 \pi)$. By definition of the limit from the ellipsoid to prolate spheroid as " $\lim _{e \rightarrow p}$ " (no need to define a limit for the oblate spheroid, since it is taken by the simple transformation, mentioned above) and to sphere as " $\lim _{e \rightarrow s}$ ", we can recover the following relations as our 3D system degenerates to the prolate spheroidal and the spherical one, respectively; those are

$$
\begin{aligned}
& \lim _{e \rightarrow p} \rho=c \tau \quad \text { with } c>0, \\
& \lim _{e \rightarrow s} \rho=r
\end{aligned}
$$

$$
\text { for every } \rho \in\left[h_{2},+\infty\right), \tau \in[1,+\infty), r \in[0,+\infty)
$$

for the radial dependence, while for every $\mu \in\left[h_{3}, h_{2}\right], \nu \in$ $\left[0, h_{3}\right]$, and $\zeta \in[-1,1]$,

$$
\begin{aligned}
& \lim _{e \rightarrow p} \frac{\mu \nu}{h_{2} h_{3}}=\lim _{e \rightarrow s} \frac{\mu \nu}{h_{2} h_{3}}=\zeta, \\
& \lim _{e \rightarrow p} \frac{\sqrt{\mu^{2}-h_{3}^{2}} \sqrt{h_{3}^{2}-\nu^{2}}}{h_{1} h_{3}}=\lim _{e \rightarrow s} \frac{\sqrt{\mu^{2}-h_{3}^{2}} \sqrt{h_{3}^{2}-\nu^{2}}}{h_{1} h_{3}} \\
& =\sqrt{1-\zeta^{2}} \cos \varphi, \\
& \lim _{e \rightarrow p} \frac{\sqrt{h_{2}^{2}-\mu^{2}} \sqrt{h_{2}^{2}-v^{2}}}{h_{1} h_{2}}=\lim _{e \rightarrow s} \frac{\sqrt{h_{2}^{2}-\mu^{2}} \sqrt{h_{2}^{2}-\nu^{2}}}{h_{1} h_{2}} \\
& =\sqrt{1-\zeta^{2}} \sin \varphi
\end{aligned}
$$

provides us with the angular dependences. To conclude, the elliptic integrals (44) become

$$
\begin{aligned}
& \lim _{e \rightarrow p} I_{\ell}^{m}(\rho)=\int_{c \tau}^{+\infty} \frac{d u}{\left[\lim _{e \rightarrow p} E_{\ell}^{m}(u)\right]^{2}\left|u^{2}-\alpha_{1}^{2}+\alpha_{2}^{2}\right|} \\
& \quad \text { with } c>0, \\
& \lim _{\ell \rightarrow s} I_{\ell}^{m}(\rho)=\frac{1}{(2 \ell+1) r^{2 \ell+1}}
\end{aligned}
$$

for $\ell \geq 0$ and $m=1,2, \ldots, 2 \ell+1$, implying

$$
\begin{aligned}
& \lim _{e \rightarrow p} I_{0}^{1}(\rho)=\frac{1}{2 c} \ln \frac{\tau+1}{\tau-1} \quad \text { with } c>0, \\
& \lim _{e \rightarrow s} I_{0}^{1}(\rho)=\frac{1}{r}
\end{aligned}
$$

for every $\rho \in\left[h_{2},+\infty\right), \tau \in[1,+\infty)$, and $r \in[0,+\infty)$. Information gathered from relation (29) up to (53) will be used extensively to our forthcoming analysis, while the geometrical and mathematical reduction that was described in between (54) and (57) was interpreted for completeness.

\section{Ellipsoidal Low-Frequency Electromagnetic Fields}

We intend to derive as handy as possible closed analytical forms as full series expansions for the surviving scattered electromagnetic fields $\mathbf{H}_{0}^{s}, \mathbf{H}_{2}^{s}, \mathbf{H}_{3}^{s}$, and $\mathbf{E}_{2}^{s}$, since $\mathbf{H}_{1}^{s}=\mathbf{E}_{0}^{s}=$ $\mathbf{E}_{1}^{s}=\mathbf{E}_{3}^{s}=\mathbf{0}$, from which the already known spherical results are readily recovered and in the sequel we wish to provide the necessary data of a representative application, concerning Earth's electromagnetic probing. To achieve it, we must independently solve problems (20) and (22) to get $\mathbf{H}_{0}^{s}$ and $\mathbf{H}_{3}^{s}$, respectively, and then proceed to problem (21) to evaluate $\mathbf{H}_{2}^{s}$ and, thus, $\mathbf{E}_{2}^{s}$, which is much more complicated due to coupling with (20). Those boundary value problems are completed by the perfectly reflecting boundary 
conditions for the total electromagnetic fields (3), given by (26) (accompanied also by the proper behavior at infinity, as (28) indicates), applied on the surface $\rho=\rho_{s} \equiv \alpha_{1}$ of the metal ellipsoid, which we conveniently choose to match the surface of the reference ellipsoid. The external scattering ellipsoidal domain is depicted by

$$
\begin{gathered}
V\left(\mathbb{R}^{3}\right)-\left\{\mathbf{r}_{0}\right\}=\left\{(\rho, \mu, \nu): \rho \in\left[\rho_{s} \equiv \alpha_{1},+\infty\right), \mu\right. \\
\left.\quad \in\left[h_{3}, h_{2}\right], \nu \in\left[0, h_{3}\right]\right\}-\left\{\left(\rho_{0}, \mu_{0}, \nu_{0}\right)\right\},
\end{gathered}
$$

in which the low-frequency magnetic and the electric fields must be built at each $n=0,2,3$, while we recall that there are no electromagnetic fields inside the ellipsoidal body. Since the actual region of observation is outside the ellipsoidal target under consideration, we use only the exterior harmonic eigenfunctions (46) for the potential problems. We start from the easiest case $n=3$, continue to $n=0$, and conclude with the most cumbersome case $n=2$.

This contribution offers a generalization of the results obtained in [5] for the particular physical application, but using the theory of ellipsoidal harmonics until a certain order $\ell=0,1,2,3$ and with $m=1,2, \ldots, 2 \ell+1$ as the degree. The reason for this constraint to the order was that only these few harmonic eigenfunctions were known in a closed-type analytical fashion [2]. Hence, in the aim of obtaining analytical results ready to accept further numerical implementation, the authors in [5] had to limit themselves to a particular number of ellipsoidal harmonics. Here, we provide a generalization of [5], which is the basis of a possible application of a new numerical method that could extend the range of the order up to very high values. However, in order to apply this unique technique, we are obliged to solve the physical and mathematical problem introduced in this work from the beginning, since we wish to insert all the orders, thus the corresponding degrees, of the ellipsoidal harmonic eigenfunctions, mentioned above, that is, for $\ell=0,1,2, \ldots$ and $m=1,2, \ldots, 2 \ell+1$, in the series expansions of the potentials. To this end, we proceed as follows.

4.1. The $\mathbf{H}_{3}^{s}$ Magnetic Field. The simplest calculations concern the scattered magnetic field $\mathbf{H}_{3}^{s}$, since the incident field (13) for $n=3$ is constant. Here, we have to solve the potential boundary value problem (22) with the Neumann boundary condition (25) on $S$ for $n=3$, which in terms of the unit normal vector $\widehat{\mathbf{n}}$ in ellipsoidal coordinates (39) is

$$
\begin{aligned}
\mathbf{H}_{3}^{s} & =\nabla \Phi_{3}^{s} \quad \text { with } \Delta \Phi_{3}^{s}=0, \\
\text { where } \widehat{\boldsymbol{\rho}} \cdot\left(\mathbf{H}_{3}^{i}+\mathbf{H}_{3}^{s}\right) & =0 \text { on } \rho=\rho_{s}=\alpha_{1} .
\end{aligned}
$$

Then, using expansion (50) with (46), the exterior harmonic structure of the potential $\Phi_{3}^{s}$ yields

$$
\begin{aligned}
\mathbf{H}_{3}^{s}= & \sum_{\ell=0}^{\infty} \sum_{m=1}^{2 \ell+1} a_{\ell}^{m} \nabla \mathbb{F}_{\ell}^{m} \\
= & \sum_{\ell=0}^{\infty} \sum_{m=1}^{2 \ell+1}(2 \ell+1) a_{\ell}^{m} \nabla\left[I_{\ell}^{m}(\rho) E_{\ell}^{m}(\rho) S_{\ell}^{m}(\mu, \nu)\right] \\
& \text { for every } \mathbf{r} \in V\left(\mathbb{R}^{3}\right)-\left\{\mathbf{r}_{0}\right\},
\end{aligned}
$$

where $a_{\ell}^{m}$ for $\ell \geq 0$ and $m=1,2, \ldots, 2 \ell+1$ stand for the constant coefficients to be determined. Thus, in terms of the primary field (13), in view of the unit dyadic, and taking the three projections of the magnetic dipole in Cartesian coordinates from (2), the condition (59), the gradient operator (36), and the unit normal vector (39) in ellipsoidal coordinates, we apply orthogonality of the surface ellipsoidal harmonics $S_{\ell}^{m}(\mu, \nu)=E_{\ell}^{m}(\mu) E_{\ell}^{m}(\nu)$ for $\ell \geq 0$ and $m=1,2, \ldots, 2 \ell+1$. The type of the incident field (13) offers nonzero constant coefficients of the solution of the field only for $S_{1}^{\kappa}(\mu, \nu)=E_{1}^{\kappa}(\mu) E_{1}^{\kappa}(\nu)$ with $\kappa=1,2,3$, as indicated by the orthogonality property (48). Therein, we come up with the expression

$$
\begin{array}{r}
\mathbf{H}_{3}^{s}=\sum_{j=1}^{3} a_{1}^{j} \nabla \mathbb{F}_{1}^{j}=3 \sum_{j=1}^{3} a_{1}^{j} \nabla\left[I_{1}^{j}(\rho) E_{1}^{j}(\rho) E_{1}^{j}(\mu) E_{1}^{j}(\nu)\right] \\
\text { for every } \mathbf{r} \in V\left(\mathbb{R}^{3}\right)-\left\{\mathbf{r}_{0}\right\},
\end{array}
$$

where

$$
a_{1}^{\kappa}=\frac{m_{\kappa}}{18 \pi} \frac{\alpha_{1} \alpha_{2} \alpha_{3}}{\left(\alpha_{1} \alpha_{2} \alpha_{3} I_{1}^{\kappa}\left(\alpha_{1}\right)-1\right)} \frac{h_{\kappa}}{h_{1} h_{2} h_{3}}
$$

for $\kappa=1,2,3$,

which is an immediate consequence of definition (31) for $x_{\kappa}$ with $\kappa=1,2,3$.

4.2. The $\mathbf{H}_{0}^{\mathcal{s}}$ Magnetic Field. Following the same procedure, we are ready to obtain the scattered field when $n=0$, that is, the static term $\mathbf{H}_{0}^{s}$, though not easy as a consequence of the complexity of the incident field $\mathbf{H}_{0}^{i}$, given by (11). This field involves double action of the gradient operator (at $\mathbf{r} \neq$ $\mathbf{r}_{0}$ ) on the quantity $1 / R$ for $R=\left|\mathbf{r}-\mathbf{r}_{0}\right|$. Therefore, we are confronted once more with a potential boundary value problem of the form (20) and we also apply the Neumann boundary condition (25) on $S$ for $n=0$, whereas for the unit normal vector $\widehat{\mathbf{n}}$ defined in ellipsoidal coordinates by (39), it is stated by

$$
\mathbf{H}_{0}^{s}=\nabla \Phi_{0}^{s} \quad \text { with } \Delta \Phi_{0}^{s}=0,
$$

where $\widehat{\boldsymbol{\rho}} \cdot\left(\mathbf{H}_{0}^{i}+\mathbf{H}_{0}^{s}\right)=0 \quad$ on $\rho=\rho_{s}=\alpha_{1}$.

Similarly with the previous analysis, the exterior harmonic potential $\Phi_{0}^{s}$ gives

$$
\begin{aligned}
\mathbf{H}_{0}^{s}= & \sum_{\ell=0}^{\infty} \sum_{m=1}^{2 \ell+1} b_{\ell}^{m} \nabla \mathbb{F}_{\ell}^{m} \\
= & \sum_{\ell=0}^{\infty} \sum_{m=1}^{2 \ell+1}(2 \ell+1) b_{\ell}^{m} \nabla\left[I_{\ell}^{m}(\rho) E_{\ell}^{m}(\rho) S_{\ell}^{m}(\mu, \nu)\right] \\
& \quad \text { for every } \mathbf{r} \in V\left(\mathbb{R}^{3}\right)-\left\{\mathbf{r}_{0}\right\},
\end{aligned}
$$

where for $\ell \geq 0$ and $m=1,2, \ldots, 2 \ell+1$ we have $S_{\ell}^{m}(\mu, \nu)=$ $E_{\ell}^{m}(\mu) E_{\ell}^{m}(\nu)$, while $b_{\ell}^{m}$ denote the constant coefficients to be 
deduced from boundary condition (63). Initially, we calculate the two parts of the condition separately. Then, in view of expression for the gradient operator in ellipsoidal coordinates (36), we come up with

$$
\begin{aligned}
\left.\widehat{\boldsymbol{\rho}} \cdot \mathbf{H}_{0}^{s}\right|_{\rho=\rho_{s}}=\frac{1}{\left.h_{\rho}\right|_{\rho=\rho_{s}}} \sum_{\ell=0}^{\infty} \sum_{m=1}^{2 \ell+1}(2 \ell+1) b_{\ell}^{m} \\
\cdot \frac{\left(\alpha_{2} \alpha_{3} E_{\ell}^{m}\left(\alpha_{1}\right) E_{\ell}^{m^{\prime}}\left(\alpha_{1}\right) I_{\ell}^{m}\left(\alpha_{1}\right)-1\right)}{\alpha_{2} \alpha_{3} E_{\ell}^{m}\left(\alpha_{1}\right)} E_{\ell}^{m}(\mu) \\
\cdot E_{\ell}^{m}(\nu),
\end{aligned}
$$

where the prime denotes derivation with respect to the argument. Yet, the expression of the incident field appears not easily amenable to further processing and an alternative approach is followed, which is the key to calculation of $\mathbf{H}_{0}^{s}$. Therein, we avoid applying the operator $\nabla \equiv \nabla_{\mathbf{r}}$ twice on $1 / R$, as indicated by relationship (11), and we first evaluate the inner product $\widehat{\rho} \cdot \nabla$ to obtain

$$
\left.\widehat{\boldsymbol{\rho}} \cdot \mathbf{H}_{0}^{i}\right|_{\rho=\rho_{s}}=\frac{1}{\left.h_{\rho}\right|_{\rho=\rho_{s}}}\left[\frac{\partial}{\partial \rho}\left(\nabla_{\mathbf{r}} \frac{1}{\left|\mathbf{r}-\mathbf{r}_{0}\right|}\right)\right]_{\rho=\rho_{s}} \cdot \frac{\mathbf{m}}{4 \pi}
$$

$$
\text { for every } \mathbf{r} \in V\left(\mathbb{R}^{3}\right)-\left\{\mathbf{r}_{0}\right\} \text {, }
$$

since the dyadic $\nabla \otimes \nabla(1 / R)$ is symmetric, while the doublederivation over quantity $1 / R$ is avoided from (15). Thus, (66) is rewritten as

$$
\left.\widehat{\boldsymbol{\rho}} \cdot \mathbf{H}_{0}^{i}\right|_{\rho=\rho_{s}}=-\frac{1}{\left.h_{\rho}\right|_{\rho=\rho_{s}}}\left[\frac{\partial}{\partial \rho}\left(\nabla_{\mathbf{r}_{0}} \frac{1}{\left|\mathbf{r}-\mathbf{r}_{0}\right|}\right)\right]_{\rho=\rho_{s}} \cdot \frac{\mathbf{m}}{4 \pi}
$$

$$
\text { for every } \mathbf{r} \in V\left(\mathbb{R}^{3}\right)-\left\{\mathbf{r}_{0}\right\} \text {, }
$$

which, given all the orthonormalization constants $\gamma_{\ell}^{m}$ for $\ell \geq$ 0 and $m=1,2, \ldots, 2 \ell+1$ from (49) and upon introduction of the proper eigenexpansion for $\rho<\rho_{0}$ via Heine's relation (53) as

$$
\begin{aligned}
\frac{1}{R} \equiv & \frac{1}{\left|\mathbf{r}-\mathbf{r}_{0}\right|} \\
= & \sum_{\ell=0}^{\infty} \sum_{m=1}^{2 \ell+1} \frac{4 \pi}{(2 \ell+1) \gamma_{\ell}^{m}} \mathbb{F}_{\ell}^{m}\left(\mathbf{r}_{0}\right) E_{\ell}^{m}(\rho) E_{\ell}^{m}(\mu) E_{\ell}^{m}(\nu) \\
& \quad \text { for every } \mathbf{r} \in V\left(\mathbb{R}^{3}\right)-\left\{\mathbf{r}_{0}\right\},
\end{aligned}
$$

becomes

$$
\begin{aligned}
\hat{\boldsymbol{\rho}} & \left.\cdot \mathbf{H}_{0}^{i}\right|_{\rho=\rho_{s}} \\
= & -\frac{1}{\left.h_{\rho}\right|_{\rho=\rho_{s}}}\left[\sum_{\ell=0}^{\infty} \sum_{m=1}^{2 \ell+1} \frac{E_{\ell}^{m^{\prime}}\left(\alpha_{1}\right)}{(2 \ell+1) \gamma_{\ell}^{m}}\left(\frac{\mathbf{m}}{4 \pi} \cdot \nabla_{\mathbf{r}_{0}} \mathbb{F}_{\ell}^{m}\left(\mathbf{r}_{0}\right)\right)\right] \\
& \cdot E_{\ell}^{m}(\mu) E_{\ell}^{m}(\nu)
\end{aligned}
$$

for $\mathbf{r} \in V\left(\mathbb{R}^{3}\right)-\left\{\mathbf{r}_{0}\right\}$. The gradient $\nabla_{\mathbf{r}_{0}} \mathbb{F}_{\ell}^{m}\left(\mathbf{r}_{0}\right)$ is a known quantity at $\mathbf{r}=\mathbf{r}_{0}$, while the magnetic dipole $\mathbf{m}$ decomposes as shown in (2). Hence, we achieved the reduction of the difficulty of boundary condition (63) by using this technique. Combining now (65) and (69), in view of (63), we obtain the unknown constant coefficients, when orthogonality of the surface ellipsoidal harmonics $E_{\ell}^{m}(\mu) E_{\ell}^{m}(\nu)$ for $\ell \geq 0$ and $m=1,2, \ldots, 2 \ell+1$ is applied through (48). Consequently, our field is given by (64) with

$$
\begin{aligned}
b_{\ell}^{m} & =\frac{1}{(2 \ell+1)^{2} \gamma_{\ell}^{m}} \\
& \cdot \frac{\alpha_{2} \alpha_{3} E_{\ell}^{m}\left(\alpha_{1}\right) E_{\ell}^{m^{\prime}}\left(\alpha_{1}\right)}{\left(\alpha_{2} \alpha_{3} E_{\ell}^{m}\left(\alpha_{1}\right) E_{\ell}^{m^{\prime}}\left(\alpha_{1}\right) I_{\ell}^{m}\left(\alpha_{1}\right)-1\right)}\left(\frac{\mathbf{m}}{4 \pi}\right. \\
& \left.\cdot \nabla_{\mathbf{r}_{0}} \mathbb{F}_{\ell}^{m}\left(\mathbf{r}_{0}\right)\right) \quad \text { for } \ell \geq 0, m=1,2, \ldots, 2 \ell+1
\end{aligned}
$$

and this field has also been calculated in a convenient and easy-to-handle closed form.

4.3. The $\mathbf{H}_{2}^{s}$ Magnetic and $\mathbf{E}_{2}^{s}$ Electric Fields. Let us concentrate now upon the potential problem at $n=2$, where a very cumbersome manipulation of the boundary value problem (21) with (25) results in the dynamic scattered fields $\mathbf{H}_{2}^{s}$ and $\mathbf{E}_{2}^{s}$. There exist two reasons for this difficulty. The first one is the coupling of the particular model with the zeroorder field $\mathbf{H}_{0}^{s}$ (static term) and the second one refers to the extra electric field $\mathbf{E}_{2}^{s}$, which enters with the corresponding additional boundary conditions. However, $\mathbf{H}_{2}^{s}$ as well as $\mathbf{E}_{2}^{s}$ terms are of major significance, since they provide purely imaginary-valued field components within the conductive medium, as seen from (18) and (19), and contribute to at least most of the imaginary-valued (quadrature) part of the magnetic field $\mathbf{H}^{s}$ and to the entire imaginary term of the corresponding electric field $\mathbf{E}^{s}$. Indeed, the real-valued (inphase) part of $\mathbf{H}^{s}$ is essentially made of the static contribution $\mathbf{H}_{0}^{s}$. The mathematical problem to solve is summarized by (21) and (25), which, in terms of the normal unit vector $\hat{\boldsymbol{\rho}}$ in ellipsoidal coordinates (see (39)), becomes

$$
\begin{aligned}
\mathbf{H}_{2}^{s}=\boldsymbol{\Phi}_{2}^{s} & +\frac{1}{2}\left(\mathbf{r} \Phi_{0}^{s}\right) \\
& \text { with } \Delta \Phi_{2}^{s}=\mathbf{0}, \Delta \Phi_{0}^{s}=0,
\end{aligned}
$$

where $\widehat{\boldsymbol{\rho}} \cdot\left(\mathbf{H}_{2}^{i}+\mathbf{H}_{2}^{s}\right)=0 \quad$ on $\rho=\rho_{s}=\alpha_{1}$,

$$
\begin{aligned}
\mathbf{E}_{2}^{s} & =\frac{1}{\sigma} \nabla \times \mathbf{H}_{2}^{s} \\
& =\frac{1}{\sigma}\left(\nabla \times \Phi_{2}^{s}+\frac{1}{2} \nabla \Phi_{0}^{s} \times \mathbf{r}\right),
\end{aligned}
$$

where $\hat{\boldsymbol{\rho}} \times\left(\mathbf{E}_{2}^{i}+\mathbf{E}_{2}^{s}\right)=\mathbf{0} \quad$ on $\rho=\rho_{s}=\alpha_{1}$,

where the second equality for the scattered electric field in (38) comes from the application of a trivial identity. Even though the divergence-free character of $\mathbf{E}_{2}^{\mathcal{S}}$ is obvious, this 
is not the case for the scattered magnetic field $\mathbf{H}_{2}^{s}$, where we have

$$
\begin{aligned}
\nabla \cdot \mathbf{H}_{2}^{s}=0 \Longrightarrow 2 \nabla \cdot \Phi_{2}^{s} & +3 \Phi_{0}^{s}+\left(\mathbf{r} \cdot \nabla \Phi_{0}^{s}\right)=0 \\
& \text { for every } \mathbf{r} \in V\left(\mathbb{R}^{3}\right)-\left\{\mathbf{r}_{0}\right\},
\end{aligned}
$$

as a consequence of the direct application of another trivial identity onto (71) using $\nabla \cdot \mathbf{r}=3$. Result (73) stands for the extra condition that must be satisfied in addition to the three (one scalar and two components of a vector) boundary conditions in (71) and (72). The coupling with the Rayleigh approximation solution at $n=0$ is exhibited for the nonharmonic part of the field $\mathbf{H}_{2}^{s}$.

Hence, in terms of the already calculated constant coefficients $b_{\ell}^{m}$ for $\ell \geq 0$ and $m=1,2, \ldots, 2 \ell+1$ in (70), we write the potential $\Phi_{0}^{s}$ as

$$
\begin{aligned}
\Phi_{0}^{s} & =\sum_{\ell=0}^{\infty} \sum_{m=1}^{2 \ell+1} b_{\ell}^{m} \mathbb{F}_{\ell}^{m} \\
= & \sum_{\ell=0}^{\infty} \sum_{m=1}^{2 \ell+1}(2 \ell+1) b_{\ell}^{m}\left[I_{\ell}^{m}(\rho) E_{\ell}^{m}(\rho)\right] S_{\ell}^{m}(\mu, \nu) \\
& \text { for every } \mathbf{r} \in V\left(\mathbb{R}^{3}\right)-\left\{\mathbf{r}_{0}\right\} .
\end{aligned}
$$

Additionally, the second set of functions of the dynamic field $\mathbf{H}_{2}^{s}$ is built up from the harmonic character of $\boldsymbol{\Phi}_{2}^{s}$ for external (outside the given ellipsoid) domains, that is, by virtue of (50) with (46),

$$
\begin{aligned}
\boldsymbol{\Phi}_{2}^{s} & =\sum_{\ell=0}^{\infty} \sum_{m=1}^{2 \ell+1} \mathbf{c}_{\ell}^{m} \mathbb{F}_{\ell}^{m} \\
= & \sum_{\ell=0}^{\infty} \sum_{m=1}^{2 \ell+1}(2 \ell+1) \mathbf{c}_{\ell}^{m}\left[I_{\ell}^{m}(\rho) E_{\ell}^{m}(\rho)\right] S_{\ell}^{m}(\mu, \nu) \\
& \text { for every } \mathbf{r} \in V\left(\mathbb{R}^{3}\right)-\left\{\mathbf{r}_{0}\right\},
\end{aligned}
$$

where

$$
\begin{aligned}
\mathbf{c}_{\ell}^{m}=c_{\ell, 1}^{m} \widehat{\mathbf{x}}_{1}+c_{\ell, 2}^{m} \widehat{\mathbf{x}}_{2}+c_{\ell, 3}^{m} \widehat{\mathbf{x}}_{3} & =\sum_{j=1}^{3} c_{\ell, j}^{m} \widehat{\mathbf{x}}_{j} \\
& \text { for } \ell \geq 0, m=1,2, \ldots, 2 \ell+1
\end{aligned}
$$

denote the vector character of the unknown constant coefficients $\mathbf{c}_{\ell}^{m}$, given through the scalar ones for $\ell \geq 0$ and $m=$ $1,2, \ldots, 2 \ell+1$. Thus, according to expansions (74) and (75) and in terms of the ellipsoidal representation of the position vector

$$
\begin{aligned}
\mathbf{r}=\sum_{j=1}^{3} x_{j} \widehat{\mathbf{x}}_{j}=\frac{\rho}{h_{\rho}} \widehat{\boldsymbol{\rho}}+\frac{\mu}{h_{\mu}} \widehat{\boldsymbol{\mu}}+\frac{\nu}{h_{\nu}} \widehat{\boldsymbol{\nu}} & \\
& \text { for every } \mathbf{r} \in V\left(\mathbb{R}^{3}\right)-\left\{\mathbf{r}_{0}\right\},
\end{aligned}
$$

the full solution (71) for the scattered magnetic field $\mathbf{H}_{2}^{s}$ is expressed as

$$
\begin{gathered}
\mathbf{H}_{2}^{s}=\sum_{\ell=0}^{\infty} \sum_{m=1}^{2 \ell+1}\left(\mathbf{c}_{\ell}^{m}+\frac{b_{\ell}^{m}}{2} \mathbf{r}\right) \mathbb{F}_{\ell}^{m}=\sum_{\ell=0}^{\infty} \sum_{m=1}^{2 \ell+1}(2 \ell+1) \\
\cdot\left(\mathbf{c}_{\ell}^{m}+\frac{b_{\ell}^{m}}{2} \mathbf{r}\right)\left[I_{\ell}^{m}(\rho) E_{\ell}^{m}(\rho)\right] S_{\ell}^{m}(\mu, \nu)
\end{gathered}
$$

for every $\mathbf{r} \in V\left(\mathbb{R}^{3}\right)-\left\{\mathbf{r}_{0}\right\}$, while the scattered electric field (72) is assumed to be

$$
\begin{aligned}
\mathbf{E}_{2}^{s} & =\frac{1}{\sigma} \sum_{\ell=0}^{\infty} \sum_{m=1}^{2 \ell+1} \nabla \mathbb{F}_{\ell}^{m} \times\left(\mathbf{c}_{\ell}^{m}+\frac{b_{\ell}^{m}}{2} \mathbf{r}\right)=\frac{1}{\sigma} \\
& \cdot \sum_{\ell=0}^{\infty} \sum_{m=1}^{2 \ell+1}(2 \ell+1) \nabla\left[I_{\ell}^{m}(\rho) E_{\ell}^{m}(\rho) S_{\ell}^{m}(\mu, \nu)\right] \\
& \times\left(\mathbf{c}_{\ell}^{m}+\frac{b_{\ell}^{m}}{2} \mathbf{r}\right)
\end{aligned}
$$

for every $\mathbf{r} \in V\left(\mathbb{R}^{3}\right)-\left\{\mathbf{r}_{0}\right\}$, by straightforward application of an identity, since $\nabla \times \mathbf{r}=\mathbf{0}$. In this part of the calculations we have to work within the frame of purely ellipsoidal geometry. Thus, we represent the fields (78) and (79) in ellipsoidal coordinates and to accomplish that we introduce the ellipsoidal image of the constant coefficients $\mathbf{c}_{\ell}^{m}$ from (76), which for the prescribed $\mathbf{r} \in V\left(\mathbb{R}^{3}\right)-\left\{\mathbf{r}_{0}\right\}$ is

$$
\begin{aligned}
\mathbf{c}_{\ell}^{m} & =\sum_{j=1}^{3} c_{\ell, j}^{m} x_{j}\left(\frac{\rho}{h_{\rho}} \frac{\widehat{\boldsymbol{\rho}}}{\rho^{2}-\alpha_{1}^{2}+\alpha_{j}^{2}}+\frac{\mu}{h_{\mu}} \frac{\widehat{\boldsymbol{\mu}}}{\mu^{2}-\alpha_{1}^{2}+\alpha_{j}^{2}}\right. \\
& \left.+\frac{\nu}{h_{\nu}} \frac{\widehat{\boldsymbol{\nu}}}{v^{2}-\alpha_{1}^{2}+\alpha_{j}^{2}}\right)
\end{aligned}
$$

$$
\text { for } \ell \geq 0, m=1,2, \ldots, 2 \ell+1 \text {. }
$$

In order to derive expression (80), we used the unit normal vectors of system (38). The three sets $c_{\ell, \kappa}^{m}$ of scalar constant coefficients for $\kappa=1,2,3$ introduced above must be calculated in accordance with the incident field data (12) and (14), with the particular solution (21) and boundary conditions (71) and (72), provided that condition (73) is automatically ensured. It is obvious that the field problem for $n=2$ has additional difficulty, inherited from the corresponding mathematical complexity. Thus, the analytical procedure for the evaluation of the unknown constant coefficients is considered below and depicts only the main steps, due to the large amount of performed calculations.

Before that, it would be helpful to apply the general condition (73) on the surface of the ellipsoid $\rho=\rho_{s}=$ $\alpha_{1}$; that is, for $S_{\ell}^{m}(\mu, \nu)=E_{\ell}^{m}(\mu) E_{\ell}^{m}(\nu)$ with $\ell \geq 0$ and 
$m=1,2, \ldots, 2 \ell+1$, we reach the following reinforced condition; that is,

$$
\begin{aligned}
& \sum_{\ell=0}^{\infty} \sum_{m=1}^{2 \ell+1}(2 \ell+1) E_{\ell}^{m}\left(\alpha_{1}\right) I_{\ell}^{m}\left(\alpha_{1}\right) E_{\ell}^{m}(\mu) E_{\ell}^{m}(\nu) \\
& .\left\{b _ { \ell } ^ { m } \left[3+\frac{\alpha_{1} \alpha_{2} \alpha_{3}}{\left(\alpha_{1}^{2}-\mu^{2}\right)\left(\alpha_{1}^{2}-v^{2}\right)}\right.\right. \\
& . \frac{\left(\alpha_{2} \alpha_{3} E_{\ell}^{m}\left(\alpha_{1}\right) E_{\ell}^{m^{\prime}}\left(\alpha_{1}\right) I_{\ell}^{m}\left(\alpha_{1}\right)-1\right)}{\left[E_{\ell}^{m}\left(\alpha_{1}\right)\right]^{2} I_{\ell}^{m}\left(\alpha_{1}\right)} \\
& +\frac{1}{\left(\mu^{2}-v^{2}\right)}\left(\frac{\mu\left(\mu^{2}-h_{3}^{2}\right)\left(h_{2}^{2}-\mu^{2}\right)}{\left(\alpha_{1}^{2}-\mu^{2}\right)} \frac{E_{\ell}^{m^{\prime}}(\mu)}{E_{\ell}^{m}(\mu)}\right. \\
& \left.\left.+\frac{\nu^{m}\left(h_{3}^{2}-v^{2}\right)\left(h_{2}^{2}-v^{2}\right)}{\left(\alpha_{1}^{2}-v^{2}\right)} \frac{E_{\ell}^{m^{\prime}}(\nu)}{E_{\ell}^{m}(\nu)}\right)\right] \\
& +2 \sum_{j=1}^{3} \frac{\alpha_{1} \alpha_{2} \alpha_{3}}{\alpha_{j} h_{1} h_{2} h_{3}} E_{1}^{j}(\mu) E_{1}^{j}(\nu) \\
& \left(\alpha_{1}^{2}-\mu^{2}\right)\left(\alpha_{1}^{2}-v^{2}\right)
\end{aligned}
$$

$$
\begin{aligned}
& \frac{\left(\alpha_{2} \alpha_{3} E_{\ell}^{m}\left(\alpha_{1}\right) E_{\ell}^{m^{\prime}}\left(\alpha_{1}\right) I_{\ell}^{m}\left(\alpha_{1}\right)-1\right)}{\left[E_{\ell}^{m}\left(\alpha_{1}\right)\right]^{2} I_{\ell}^{m}\left(\alpha_{1}\right)} \\
& +\frac{\alpha_{j}^{2}}{\left(\mu^{2}-v^{2}\right)}\left(\frac{\mu\left(\mu^{2}-h_{3}^{2}\right)\left(h_{2}^{2}-\mu^{2}\right)}{\left(\mu^{2}-\alpha_{1}^{2}+\alpha_{j}^{2}\right)\left(\alpha_{1}^{2}-\mu^{2}\right)} \frac{E_{\ell}^{m^{\prime}}(\mu)}{E_{\ell}^{m}(\mu)}\right. \\
& \left.\left.\left.+\frac{\nu\left(h_{3}^{2}-v^{2}\right)\left(h_{2}^{2}-\nu^{2}\right)}{\left(\nu^{2}-\alpha_{1}^{2}+\alpha_{j}^{2}\right)\left(\alpha_{1}^{2}-\nu^{2}\right)} \frac{E_{\ell}^{m^{\prime}}(\nu)}{E_{\ell}^{m}(\nu)}\right)\right]\right\}=0
\end{aligned}
$$

for every $\mu \in\left[h_{3}, h_{2}\right]$ and $\nu \in\left[0, h_{3}\right]$, which stands for a convenient criterion for confirmation of our results when satisfied.

We must obtain one set of relations from boundary condition (71) concerning the $\rho$-component and two sets of relations from boundary condition (72), which correspond to the $\mu, \nu$-components. Every set configuration refers to the impenetrable surface of the ellipsoidal body at $S: \rho=\rho_{s}$, which offers three boundary expressions for the coefficients given in (76). In that sense, we apply the boundary conditions (71) and (72) to the total electromagnetic fields and in view of (77)-(80), in terms of expansion (68), as well as in view of the second-equality form of the incident fields (12) and (14), we reach in cumbersome fashion the following three relations for the constant coefficients, where we have used extensively (43)-(47). Initially, from (71) we conclude that

$$
\begin{aligned}
& \sum_{\ell=0}^{\infty} \sum_{m=1}^{2 \ell+1} E_{\ell}^{m}(\mu) E_{\ell}^{m}(\nu) \\
& \cdot\left\{\sum_{j=1}^{3} \frac{h_{j} E_{1}^{j}\left(\alpha_{1}\right)}{h_{1} h_{2} h_{3}} E_{1}^{j}(\mu) E_{1}^{j}(\nu)\left[\frac{2(2 \ell+1) I_{\ell}^{m}\left(\alpha_{1}\right) E_{\ell}^{m}\left(\alpha_{1}\right)}{\alpha_{j}^{2}} c_{\ell, j}^{m}+\frac{\mathbb{F}_{\ell}^{m}\left(\mathbf{r}_{0}\right)}{(2 \ell+1) \gamma_{\ell}^{m}}\left(\frac{E_{\ell}^{m^{\prime}}\left(\alpha_{1}\right)}{\alpha_{1}}-\frac{E_{\ell}^{m}\left(\alpha_{1}\right)}{\alpha_{j}^{2}}\right) \frac{m_{j}}{4 \pi}\right]\right. \\
& \left.+\left[(2 \ell+1) I_{\ell}^{m}\left(\alpha_{1}\right) E_{\ell}^{m}\left(\alpha_{1}\right) b_{\ell}^{m}-\frac{\mathbb{F}_{\ell}^{m}\left(\mathbf{r}_{0}\right)}{(2 \ell+1) \gamma_{\ell}^{m}} \frac{E_{\ell}^{m^{\prime}}\left(\alpha_{1}\right)}{\alpha_{1}}\left(\frac{\mathbf{m}}{4 \pi} \cdot \mathbf{r}_{0}\right)\right]\right\}=0
\end{aligned}
$$

for every value of $\mu \in\left[h_{3}, h_{2}\right]$ and $\nu \in\left[0, h_{3}\right]$, concerning the $\rho$-component of boundary condition (71), where we have implied the Cartesian representations of ellipsoidal harmonics of the first-order such as

$$
\begin{aligned}
x_{\kappa} & =\frac{h_{\kappa}}{h_{1} h_{2} h_{3}} \mathbb{E}_{1}^{\kappa}=\frac{h_{\kappa}}{h_{1} h_{2} h_{3}} E_{1}^{\kappa}(\rho) S_{1}^{\kappa}(\mu, \nu) \\
& =\frac{h_{\kappa}}{h_{1} h_{2} h_{3}} E_{1}^{\kappa}(\rho) E_{1}^{\kappa}(\mu) E_{1}^{\kappa}(\nu)
\end{aligned}
$$

$$
\text { for every } \mathbf{r} \in V\left(\mathbb{R}^{3}\right)-\left\{\mathbf{r}_{0}\right\} \text {. }
$$

Moreover, in view of (83), the $\mu$-component and the $\nu$ component, respectively, of condition (72) yield the complicated formulae

$$
\begin{aligned}
& \sum_{\ell=0}^{\infty} \sum_{m=1}^{2 \ell+1} E_{\ell}^{m}(\mu) E_{\ell}^{m}(\nu)\left\{\frac{(2 \ell+1)}{E_{\ell}^{m}\left(\alpha_{1}\right)}\right. \\
& \cdot \frac{\nu \sqrt{h_{3}^{2}-v^{2}} \sqrt{h_{2}^{2}-v^{2}}}{\sqrt{\mu^{2}-v^{2}} \sqrt{\alpha_{1}^{2}-\mu^{2}}\left(\alpha_{1}^{2}-v^{2}\right)}\left[\frac { b _ { \ell } ^ { m } } { 2 } \left(\alpha_{2} \alpha_{3} E_{\ell}^{m}\left(\alpha_{1}\right)\right.\right. \\
& \left.\cdot I_{\ell}^{m}\left(\alpha_{1}\right)\left(E_{\ell}^{m \prime}\left(\alpha_{1}\right)-\alpha_{1} E_{\ell}^{m}\left(\alpha_{1}\right) \frac{E_{\ell}^{m^{\prime}}(\nu)}{\nu E_{\ell}^{m}(\nu)}\right)-1\right)
\end{aligned}
$$


$+\sum_{j=1}^{3} \frac{h_{j}}{\alpha_{j} h_{1} h_{2} h_{3}} \frac{E_{1}^{j}(\mu) E_{1}^{j}(\nu)}{\left(\nu^{2}-\alpha_{1}^{2}+\alpha_{j}^{2}\right)} c_{\ell, j}^{m}$

$\cdot\left(\left(\alpha_{2} \alpha_{3} E_{\ell}^{m}\left(\alpha_{1}\right) E_{\ell}^{m^{\prime}}\left(\alpha_{1}\right) I_{\ell}^{m}\left(\alpha_{1}\right)-1\right) \alpha_{j}^{2}\right.$

$-\alpha_{1} \alpha_{2} \alpha_{3}\left[E_{\ell}^{m}\left(\alpha_{1}\right)\right]^{2} I_{\ell}^{m}\left(\alpha_{1}\right)$

$\left.\left.\cdot \frac{\left(\nu^{2}-\alpha_{1}^{2}+\alpha_{j}^{2}\right) E_{\ell}^{m^{\prime}}(\nu)}{\nu E_{\ell}^{m}(\nu)}\right)\right]-\frac{E_{\ell}^{m}\left(\alpha_{1}\right)}{(2 \ell+1) \gamma_{\ell}^{m}} \widehat{\boldsymbol{\mu}}\left(\alpha_{1}, \mu\right.$,

$\left.v) \cdot\left(\frac{\mathbf{m}}{4 \pi} \times \nabla_{\mathbf{r}_{0}} \mathbb{F}_{\ell}^{m}\left(\mathbf{r}_{0}\right)\right)\right\}=0$,

$$
\begin{aligned}
& \sum_{\ell=0}^{\infty} \sum_{m=1}^{2 \ell+1} E_{\ell}^{m}(\mu) E_{\ell}^{m}(\nu)\left\{\frac{(2 \ell+1)}{E_{\ell}^{m}\left(\alpha_{1}\right)}\right. \\
& \cdot \frac{\mu \sqrt{\mu^{2}-h_{3}^{2}} \sqrt{h_{2}^{2}-\mu^{2}}}{\sqrt{\mu^{2}-v^{2}} \sqrt{\alpha_{1}^{2}-v^{2}}\left(\alpha_{1}^{2}-\mu^{2}\right)}\left[\frac { b _ { \ell } ^ { m } } { 2 } \left(\alpha_{2} \alpha_{3} E_{\ell}^{m}\left(\alpha_{1}\right)\right.\right. \\
& \left.\cdot I_{\ell}^{m}\left(\alpha_{1}\right)\left(E_{\ell}^{m^{\prime}}\left(\alpha_{1}\right)-\alpha_{1} E_{\ell}^{m}\left(\alpha_{1}\right) \frac{E_{\ell}^{m^{\prime}}(\mu)}{\mu E_{\ell}^{m}(\mu)}\right)-1\right) \\
& +\sum_{j=1}^{3} \frac{h_{j}}{\alpha_{j} h_{1} h_{2} h_{3}} \frac{E_{1}^{j}(\mu) E_{1}^{j}(\nu)}{\left(\mu^{2}-\alpha_{1}^{2}+\alpha_{j}^{2}\right)} c_{\ell, j}^{m} \\
& \cdot\left(\left(\alpha_{2} \alpha_{3} E_{\ell}^{m}\left(\alpha_{1}\right) E_{\ell}^{m^{\prime}}\left(\alpha_{1}\right) I_{\ell}^{m}\left(\alpha_{1}\right)-1\right) \alpha_{j}^{2}\right. \\
& -\alpha_{1} \alpha_{2} \alpha_{3}\left[E_{\ell}^{m}\left(\alpha_{1}\right)\right]^{2} I_{\ell}^{m}\left(\alpha_{1}\right) \\
& +\left(\mu^{2}-\alpha_{1}^{2}+\alpha_{j}^{2}\right) E_{\ell}^{m^{\prime}}(\mu) \\
& +\mu E_{\ell}^{m}(\mu) \\
& \left.+\left(\frac{\mathbf{m}}{4 \pi} \times \nabla_{\mathbf{r}_{0}} \mathbb{F}_{\ell}^{m}\left(\mathbf{r}_{0}\right)\right)\right\}=\frac{E_{\ell}^{m}\left(\alpha_{1}\right)}{(2 \ell+1) \gamma_{\ell}^{m}} \widehat{\boldsymbol{v}}\left(\alpha_{1}, \mu,\right.
\end{aligned}
$$

for every value of $\mu \in\left[h_{3}, h_{2}\right]$ and $\nu \in\left[0, h_{3}\right]$. The unit normal vectors defined in (38), being given via

$$
\begin{aligned}
& \widehat{\boldsymbol{\mu}}\left(\alpha_{1}, \mu, \nu\right) \\
& =\frac{\mu \sqrt{\mu^{2}-h_{3}^{2}} \sqrt{h_{2}^{2}-\mu^{2}}}{\sqrt{\mu^{2}-v^{2}} \sqrt{\alpha_{1}^{2}-\mu^{2}}} \sum_{j=1}^{3} \frac{h_{j} E_{1}^{j}\left(\alpha_{1}\right)}{h_{1} h_{2} h_{3}} \frac{E_{1}^{j}(\mu) E_{1}^{j}(\nu)}{\mu^{2}-\alpha_{1}^{2}+\alpha_{j}^{2}} \widehat{\mathbf{x}}_{j}, \\
& \widehat{\boldsymbol{v}}\left(\alpha_{1}, \mu, \nu\right) \\
& =\frac{\nu \sqrt{h_{3}^{2}-v^{2}} \sqrt{h_{2}^{2}-v^{2}}}{\sqrt{\mu^{2}-v^{2}} \sqrt{\alpha_{1}^{2}-v^{2}}} \sum_{j=1}^{3} \frac{h_{j} E_{1}^{j}\left(\alpha_{1}\right)}{h_{1} h_{2} h_{3}} \frac{E_{1}^{j}(\mu) E_{1}^{j}(\nu)}{\nu^{2}-\alpha_{1}^{2}+\alpha_{j}^{2}} \widehat{\mathbf{x}}_{j},
\end{aligned}
$$

are calculated on the surface of the ellipsoid $\rho=\rho_{s}=\alpha_{1}$, using the metric coefficients (35), as well as (83).

Relationships (82) and (84)-(85) with (86) stand for the aforementioned three boundary conditions and they form a system of three equations with the three sets of the unknown constant coefficients $c_{\ell, \kappa}^{m}$ for $\kappa=1,2,3$, taking into account the solution of the $b_{\ell}^{m}$ constant coefficient obtained in (70) for every $\ell \geq 0$ and $m=1,2, \ldots, 2 \ell+1$. As said, we omit many steps, which contain complicated analytical manipulations, until we reach the above expressions. These conditions are not appropriate to profit orthogonality for the surface ellipsoidal harmonics $E_{\ell}^{m}(\mu) E_{\ell}^{m}(\nu)$ for every value of $\ell \geq 0$ and $m=$ $1,2, \ldots, 2 \ell+1$, since the expressions are very complicated and, unfortunately, recurrence relations for the ellipsoidal Lamé functions do not exist. Meaning, we cannot proceed in the same manner as in the previous $n=3$ and $n=0$ cases. So, a possible numerical handling in order to obtain the three scalar constant coefficients $c_{\ell, \kappa}^{m}$ for $\kappa=1,2,3$, thus the corresponding vector ones $\mathbf{c}_{\ell}^{m}=\sum_{j=1}^{3} c_{\ell, j}^{m} \widehat{\mathbf{x}}_{j}$ for $\ell \geq 0$ and $m=1,2, \ldots, 2 \ell+1$, is inevitable. Consequently, we can evaluate the scattered magnetic field (78), satisfying the extra relation (73) and the corresponding scattered electric field (79) up to a certain level of accuracy.

However, to completely achieve analytical formulae and simplify such a numerical implementation, we avoid the difficultness arisen during the study of the three relationships (82) and (84)-(85) with (86). In order to accomplish this, we notate (82) for $\kappa=1$, (84) for $\kappa=2$ and (85) for $\kappa=3$ to rewrite these expressions, respectively, as

$$
\sum_{\ell=0}^{\infty} \sum_{m=1}^{2 \ell+1}\left[\sum_{j=1}^{3} f_{\ell, j}^{m, \kappa}(\mu, \nu) c_{\ell, j}^{m}+g_{\ell}^{m, \kappa}(\mu, \nu)\right]=0
$$

for every $\kappa=1,2,3$,

where

$$
\begin{aligned}
& f_{\ell, j}^{m, 1}(\mu, \nu)=\frac{2(2 \ell+1) h_{j} I_{\ell}^{m}\left(\alpha_{1}\right) E_{1}^{j}\left(\alpha_{1}\right) E_{\ell}^{m}\left(\alpha_{1}\right)}{\alpha_{j}^{2} h_{1} h_{2} h_{3}} \\
& \cdot E_{1}^{j}(\mu) E_{1}^{j}(\nu) E_{\ell}^{m}(\mu) E_{\ell}^{m}(\nu), \\
& f_{\ell, j}^{m, 2}(\mu, \nu)=\frac{(2 \ell+1) h_{j}}{\alpha_{j} h_{1} h_{2} h_{3} E_{\ell}^{m}\left(\alpha_{1}\right)} \\
& \cdot \frac{\sqrt{h_{3}^{2}-v^{2}} \sqrt{h_{2}^{2}-v^{2}}}{\sqrt{\mu^{2}-v^{2}} \sqrt{\alpha_{1}^{2}-\mu^{2}}\left(\alpha_{1}^{2}-v^{2}\right)\left(\nu^{2}-\alpha_{1}^{2}+\alpha_{j}^{2}\right)} \\
& \cdot E_{1}^{j}(\mu) E_{1}^{j}(\nu) E_{\ell}^{m}(\mu) E_{\ell}^{m}(\nu) \\
& \quad \times\left[\left(\alpha_{2} \alpha_{3} E_{\ell}^{m}\left(\alpha_{1}\right) E_{\ell}^{m^{\prime}}\left(\alpha_{1}\right) I_{\ell}^{m}\left(\alpha_{1}\right)-1\right) \alpha_{j}^{2}\right. \\
&- {\left[\alpha_{1} \alpha_{2} \alpha_{3}\left[E_{\ell}^{m}\left(\alpha_{1}\right)\right]^{2} I_{\ell}^{m}\left(\alpha_{1}\right)\right.} \\
&\left.\cdot \frac{\left(\nu^{2}-\alpha_{1}^{2}+\alpha_{j}^{2}\right) E_{\ell}^{m^{\prime}}(\nu)}{\nu E_{\ell}^{m}(\nu)}\right],
\end{aligned}
$$




$$
\begin{aligned}
& f_{\ell, j}^{m, 3}(\mu, v)=\frac{(2 \ell+1) h_{j}}{\alpha_{j} h_{1} h_{2} h_{3} E_{\ell}^{m}\left(\alpha_{1}\right)} \\
& \cdot \frac{\mu \sqrt{h_{3}^{2}-\mu^{2}} \sqrt{h_{2}^{2}-\mu^{2}}}{\sqrt{\mu^{2}-v^{2}} \sqrt{\alpha_{1}^{2}-v^{2}}\left(\alpha_{1}^{2}-\mu^{2}\right)\left(\mu^{2}-\alpha_{1}^{2}+\alpha_{j}^{2}\right)} \\
& \cdot E_{1}^{j}(\mu) E_{1}^{j}(\nu) E_{\ell}^{m}(\mu) E_{\ell}^{m}(\nu) \\
& \times\left[\left(\alpha_{2} \alpha_{3} E_{\ell}^{m}\left(\alpha_{1}\right) E_{\ell}^{m^{\prime}}\left(\alpha_{1}\right) I_{\ell}^{m}\left(\alpha_{1}\right)-1\right) \alpha_{j}^{2}\right. \\
& -\alpha_{1} \alpha_{2} \alpha_{3}\left[E_{\ell}^{m}\left(\alpha_{1}\right)\right]^{2} I_{\ell}^{m}\left(\alpha_{1}\right) \\
& \left.\cdot \frac{\left(\mu^{2}-\alpha_{1}^{2}+\alpha_{j}^{2}\right) E_{\ell}^{m^{\prime}}(\mu)}{\mu E_{\ell}^{m}(\mu)}\right]
\end{aligned}
$$

for every $j=1,2,3$, while $\ell \geq 0$ and $m=1,2, \ldots, 2 \ell+1$. In addition,

$$
\begin{aligned}
& g_{\ell}^{m, 1}(\mu, \nu)=E_{\ell}^{m}(\mu) E_{\ell}^{m}(\nu)\left\{\frac{\mathbb{F}_{\ell}^{m}\left(\mathbf{r}_{0}\right)}{4 \pi(2 \ell+1) \gamma_{\ell}^{m} h_{1} h_{2} h_{3}}\right. \\
& \cdot \sum_{j=1}^{3} m_{j} h_{j} E_{1}^{j}\left(\alpha_{1}\right)\left(\frac{E_{\ell}^{m^{\prime}}\left(\alpha_{1}\right)}{\alpha_{1}}-\frac{E_{\ell}^{m}\left(\alpha_{1}\right)}{\alpha_{j}^{2}}\right) E_{1}^{j}(\mu) \\
& \cdot E_{1}^{j}(v)+\left[(2 \ell+1) I_{\ell}^{m}\left(\alpha_{1}\right) E_{\ell}^{m}\left(\alpha_{1}\right) b_{\ell}^{m}\right. \\
& \left.\left.-\frac{\mathbb{F}_{\ell}^{m}\left(\mathbf{r}_{0}\right)}{(2 \ell+1) \gamma_{\ell}^{m}} \frac{E_{\ell}^{m^{\prime}}\left(\alpha_{1}\right)}{\alpha_{1}}\left(\frac{\mathbf{m}}{4 \pi} \cdot \mathbf{r}_{0}\right)\right]\right\} \\
& g_{\ell}^{m, 2}(\mu, \nu)=E_{\ell}^{m}(\mu) E_{\ell}^{m}(\nu)\left\{-\frac{E_{\ell}^{m}\left(\alpha_{1}\right)}{(2 \ell+1) \gamma_{\ell}^{m}} \widehat{\boldsymbol{\mu}}\left(\alpha_{1}, \mu, \nu\right)\right. \\
& \cdot\left(\frac{\mathbf{m}}{4 \pi} \times \nabla_{\mathbf{r}_{0}} \mathbb{F}_{\ell}^{m}\left(\mathbf{r}_{0}\right)\right)+\frac{(2 \ell+1) b_{\ell}^{m}}{2 E_{\ell}^{m}\left(\alpha_{1}\right)} \\
& \cdot \frac{\nu \sqrt{h_{3}^{2}-v^{2}} \sqrt{h_{2}^{2}-v^{2}}}{\sqrt{\mu^{2}-v^{2}} \sqrt{\alpha_{1}^{2}-\mu^{2}}\left(\alpha_{1}^{2}-v^{2}\right)}\left[\alpha_{2} \alpha_{3} E_{\ell}^{m}\left(\alpha_{1}\right)\right. \\
& \left.\left.\cdot I_{\ell}^{m}\left(\alpha_{1}\right)\left(E_{\ell}^{m^{\prime}}\left(\alpha_{1}\right)-\alpha_{1} E_{\ell}^{m}\left(\alpha_{1}\right) \frac{E_{\ell}^{m^{\prime}}(v)}{v E_{\ell}^{m}(\nu)}\right)-1\right]\right\} \text {, } \\
& g_{\ell}^{m, 3}(\mu, \nu)=E_{\ell}^{m}(\mu) E_{\ell}^{m}(\nu)\left\{\frac{E_{\ell}^{m}\left(\alpha_{1}\right)}{(2 \ell+1) \gamma_{\ell}^{m}} \widehat{\nu}\left(\alpha_{1}, \mu, \nu\right)\right. \\
& \cdot\left(\frac{\mathbf{m}}{4 \pi} \times \nabla_{\mathbf{r}_{0}} \mathbb{F}_{\ell}^{m}\left(\mathbf{r}_{0}\right)\right)+\frac{(2 \ell+1) b_{\ell}^{m}}{2 E_{\ell}^{m}\left(\alpha_{1}\right)}
\end{aligned}
$$

$$
\begin{gathered}
\cdot \frac{\mu \sqrt{h_{3}^{2}-\mu^{2}} \sqrt{h_{2}^{2}-\mu^{2}}}{\sqrt{\mu^{2}-v^{2}} \sqrt{\alpha_{1}^{2}-v^{2}}\left(\alpha_{1}^{2}-\mu^{2}\right)}\left[\alpha_{2} \alpha_{3} E_{\ell}^{m}\left(\alpha_{1}\right)\right. \\
\left.\left.\cdot I_{\ell}^{m}\left(\alpha_{1}\right)\left(E_{\ell}^{m^{\prime}}\left(\alpha_{1}\right)-\alpha_{1} E_{\ell}^{m}\left(\alpha_{1}\right) \frac{E_{\ell}^{m^{\prime}}(\mu)}{\mu E_{\ell}^{m}(\mu)}\right)-1\right]\right\}
\end{gathered}
$$

for every $\ell \geq 0$ and $m=1,2, \ldots, 2 \ell+1$. Relations (88)(89) hold true for every value of $\mu \in\left[h_{3}, h_{2}\right]$ and $\nu \in$ $\left[0, h_{3}\right]$, whereas the prime denotes derivation with respect to the argument. Hence, we can use theory (51)-(52) with (48), to expand the defined smooth ellipsoidal functions on the surface of the precise ellipsoid $\rho=\alpha_{1}$ in terms of the ellipsoidal orthonormal basis $S_{\ell}^{m}(\mu, \nu)=E_{\ell}^{m}(\mu) E_{\ell}^{m}(\nu)$ for $\ell \geq 0$ and $m=1,2, \ldots, 2 \ell+1$ as

$$
f_{\ell, j}^{m, \kappa}(\mu, \nu)=\sum_{\ell^{\prime}=0}^{\infty} \sum_{m^{\prime}=1}^{2 \ell^{\prime}+1} d_{\left(\ell, \ell^{\prime}\right), j}^{\left(m, m^{\prime}\right), \kappa} E_{\ell^{\prime}}^{m^{\prime}}(\mu) E_{\ell^{\prime}}^{m^{\prime}}(\nu)
$$

for $\ell \geq 0, m=1,2, \ldots, 2 \ell+1$, while $j, \kappa=1,2,3$,

$$
g_{\ell}^{m, \kappa}(\mu, \nu)=\sum_{\ell^{\prime}=0}^{\infty} \sum_{m^{\prime}=1}^{2 \ell^{\prime}+1} e_{\left(\ell, \ell^{\prime}\right)}^{\left(m, m^{\prime}\right), \kappa} E_{\ell^{\prime}}^{m^{\prime}}(\mu) E_{\ell^{\prime}}^{m^{\prime}}(\nu)
$$

for $\ell \geq 0, m=1,2, \ldots, 2 \ell+1$, while $\kappa=1,2,3$,

where the set of constants $d_{\left(\ell, \ell^{\prime}\right), j}^{\left(m, m^{\prime}\right), \kappa}$ and $e_{\left(\ell, \ell^{\prime}\right)}^{\left(m, m^{\prime}\right), \kappa}$ for $\ell, \ell^{\prime} \geq 0$ and $m=1,2, \ldots, 2 \ell+1, m^{\prime}=1,2, \ldots, 2 \ell^{\prime}+1$ with $j, \kappa=$ $1,2,3$ enjoy the expressions

$$
\begin{gathered}
d_{\left(\ell, \ell^{\prime}\right), j}^{\left(m, m^{\prime}\right), \kappa}=\frac{1}{\gamma_{\ell}^{m}} \iint_{\rho=\rho_{s}=\alpha_{1}} f_{\ell, j}^{m, \kappa}(\mu, \nu)\left[E_{\ell^{\prime}}^{m^{\prime}}(\mu)\right. \\
\left.\cdot E_{\ell^{\prime}}^{m^{\prime}}(\nu)\right] \frac{d S(\mu, \nu)}{\sqrt{\alpha_{1}^{2}-\mu^{2}} \sqrt{\alpha_{1}^{2}-\nu^{2}}}, \\
e_{\left(\ell, \ell^{\prime}\right)}^{\left(m, m^{\prime}\right), \kappa}=\frac{1}{\gamma_{\ell}^{m}} \iint_{\rho=\rho_{s}=\alpha_{1}} g_{\ell}^{m, \kappa}(\mu, \nu)\left[E_{\ell^{\prime}}^{m^{\prime}}(\mu)\right. \\
\left.\cdot E_{\ell^{\prime}}^{m^{\prime}}(\nu)\right] \frac{d S(\mu, \nu)}{\sqrt{\alpha_{1}^{2}-\mu^{2}} \sqrt{\alpha_{1}^{2}-\nu^{2}}},
\end{gathered}
$$

respectively, for every value of $\mu \in\left[h_{3}, h_{2}\right]$ and $\nu \in\left[0, h_{3}\right]$. In doing so, we transfer the difficulty of boundary conditions (82) and (84)-(85) with (86) to the direct calculation of integrals such as (92) and (93), which contain easy-tohandle functions. The next step involves the substitution of expansions (90) and (91) with (92) and (93) into the complicated boundary conditions (87), taking into account (88)-(89) and, then, applys orthogonality via (48), to obtain

$$
\sum_{\ell=0}^{\infty} \sum_{m=1}^{2 \ell+1}\left[\sum_{j=1}^{3} d_{\left(\ell, \ell^{\prime}\right), j}^{\left(m, m^{\prime}\right), \kappa} c_{\ell, j}^{m}+e_{\left(\ell, \ell^{\prime}\right)}^{\left(m, m^{\prime}\right), \kappa}\right]=0
$$

for $\ell^{\prime} \geq 0, m^{\prime}=1,2, \ldots, 2 \ell^{\prime}+1$, while $\kappa=1,2,3$ 
or

$$
\begin{aligned}
\sum_{\ell=0}^{\infty} \sum_{m=1}^{2 \ell+1}\left[d_{\left(\ell, \ell^{\prime}\right), 1}^{\left(m, m^{\prime}\right),} c_{\ell, 1}^{m}+d_{\left(\ell, \ell^{\prime}\right), 2}^{\left(m, m^{\prime}\right), \kappa} c_{\ell, 2}^{m}+d_{\left(\ell, \ell^{\prime}\right), 1}^{\left(m, m^{\prime}\right),} c_{\ell, 3}^{m}\right] \\
=-\sum_{\ell=0}^{\infty} \sum_{m=1}^{2 \ell+1} e_{\left(\ell, \ell^{\prime}\right)}^{\left(m, m^{\prime}\right), \kappa}
\end{aligned}
$$

$$
\text { for } \ell^{\prime} \geq 0, m^{\prime}=1,2, \ldots, 2 \ell^{\prime}+1 \text {, }
$$

where (95) are three separate conditions $(\kappa=1,2,3)$. They are standard systems of linear algebraic equations and they can be solved with cut-off techniques to obtain the unknown constant coefficients $\mathbf{c}_{\ell}^{m}=\sum_{j=1}^{3} c_{\ell, j}^{m} \widehat{\mathbf{x}}_{j}$ for $\ell \geq 0$ and $m=$ $1,2, \ldots, 2 \ell+1$. The $\mathbf{H}_{2}^{s}$ scattered magnetic field and the $\mathbf{E}_{2}^{s}$ scattered electric field follow from (78) and (79), respectively, both well-defined within the prescribed infinite domain (58).

4.4. Section's Recapitulation. Concluding, we have retrieved in an analytical fashion, ready for further numerical implementation, the low-frequency terms of the scattered magnetic and electric fields under consideration, $\mathbf{H}_{0}^{s}, \mathbf{H}_{2}^{s}, \mathbf{H}_{3}^{s}, \mathbf{E}_{1}^{s}$, and $\mathbf{E}_{3}^{s}$ in the domain $\Omega \equiv V\left(\mathbb{R}^{3}\right)-\left\{\mathbf{r}_{0}\right\}$ from well-posed boundary value problems. Hence, those can be collected and entered into the electromagnetic scattered fields (18) and (19), obtaining the fields in a compact analytical form in terms of infinite power series.

On the other hand, by virtue of the previous analysis about the reduction rules to the spheroidal or to the spherical geometry, the manipulation of our main results for the electromagnetic fields $\mathbf{H}_{0}^{s}, \mathbf{H}_{2}^{s}, \mathbf{H}_{3}^{s}, \mathbf{E}_{1}^{s}$, and $\mathbf{E}_{3}^{s}$ in the domain $\Omega \equiv V\left(\mathbb{R}^{3}\right)-\left\{\mathbf{r}_{0}\right\}$ given in (58) is a straightforward task and leads to recovering the corresponding and already known results from the literature, described earlier in the Introduction section for a spheroidal [10] and a spherical [7] nonpenetrable scatterer.

4.5. Physical Application for Future Work. The ellipsoidal coordinate system yields the appropriate environment for solving scattering problems involving ellipsoidal targets, since in the low-frequency realm we are faced with boundary value problems of Laplace's and Poisson's equations, which accepts separation of variables [2-4] in the ellipsoidal geometry. For this reason, problems similar to our case adopt this fitting system to obtain analytical results for the corresponding fields. Nevertheless, it is not always easy and feasible to pursue fully analytical solutions in closed-form fashion without the demand of computational involvement. Although the electromagnetic fields in the present investigation have been obtained for $n=0,2,3$ (there is no first-order field and higher order terms are not of substantial interest) in a closed analytical form of infinite series in terms of the ellipsoidal harmonics, they are not given in fully compact formation. Indeed, even though we deal with closed-type solutions for $n=0,3$, exhibiting infinite series expansions in terms of ellipsoidal harmonics of order $\ell=0,1,2, \ldots$ and degree $m=0,1,2, \ldots, 2 \ell+1$, this is not the case for the dynamic field at $n=2$. Therein, the constant coefficients are evaluated up to any order of controlled accuracy, not directly, but via a numerical solution of the boundary conditions or via cutoff techniques for solving quadratic systems of typical linear algebraic equations.

In order to provide all the data and the physical parameters of a real-life application for a possible forthcoming article with numerical implementation of our results, we consider the interesting situation of the identification of a large voluminous body, buried deep under the Earth's surface. We adopt the appropriate ellipsoidal geometry to describe a perfectly conducting ( $\sigma_{\text {body }} \rightarrow+\infty$ ) body with semiaxes $\alpha_{1}=75 \mathrm{~m}, \alpha_{2}=50 \mathrm{~m}$, and $\alpha_{3}=25 \mathrm{~m}$, where the results must be obtained for the measurable scattered magnetic field $\mathbf{H}^{s}$ via (18). In addition, some interesting limiting cases should be included, for example, the case of a prolate spheroidal object with semiaxes $\left(\alpha_{1}, \alpha_{2}, \alpha_{3}\right)=(75,25,24.9) \mathrm{m}$ and the case of a sphere with $\left(\alpha_{1}, \alpha_{2}, \alpha_{3}\right)=(50.1,50,49.9) \mathrm{m}$, where these values are taken in order to avoid any kind of indeterminacies within the obtained formulae. The ellipsoidal body is embedded in a homogeneous infinite space, as Earth's subsurface, of magnetic permeability $\mu \cong \mu_{0}=4 \pi \times 10^{-7} \mathrm{~F} / \mathrm{m}$ and electric conductivity $\sigma=2 \times 10^{-3} \mathrm{~S} / \mathrm{m}$. Assume that a magnetic dipole $\mathbf{m}=m_{3} \widehat{\mathbf{x}}_{3}$ of strength $m_{3}=4 \pi \times 10^{3} \mathrm{~A} \cdot \mathrm{m}^{2}$ is located nearby at $\left(x_{10}, x_{20}, x_{30}\right)=(200,0,0) \mathrm{m}$ and illuminates the ellipsoid at the low frequency of $50 \mathrm{~Hz}$, while the magnetic field is acquired along a vertical line at $\left(x_{1 p}, x_{2 p}, x_{3 p}\right)=$ $([-200,200], 200,0) \mathrm{m}$. Any future illustrations must contain the real parts and the imaginary parts of the approximated scattered magnetic field in units $[\mathrm{A} / \mathrm{m}]$.

\section{Conclusions}

Inductive electromagnetic means as currently employed in exploration of the Earth's subsurface and embedded voluminous bodies often call for intensive use, first at the modeling stage and later at the inversion stage, of simple tools of field calculation. Hence, it is of interest to elaborate powerful analytical methods, whose computational complexity is light enough for handy application, in order to solve forward and inverse electromagnetic scattering problems. The already ample library of scattering by simple shapes using analytical methods is open to accept new and useful analytical results.

The main goal of the present investigation had two directions. Primarily, we obtained an analytical approximation of the low-frequency behavior of the electromagnetic fields scattered by a perfectly conducting ellipsoid, embedded within a conductive environment (e.g., Earth's underground), which is illuminated by a magnetic dipole source, improving precedent researches. Secondary, we attained to recover the results concerning simpler shapes, such as the sphere and the spheroid, while we provided the adequate tools in a ready-to-use formulation for the application of a numerical code based on the evaluation of any order of ellipsoidal harmonic eigenfunctions, in order for someone to implement the aforementioned fields and achieve the desired accuracy.

The solution is obtained in terms of low-frequency expansions of the electric and magnetic scattered fields. The static term corresponds to the Rayleigh approximation and 
provides us with an important term of the real part of the magnetic field, while the second-order dynamic term, which address the first nonvanishing term, contributes to the main behavior of its imaginary part. The term of order three is a correction to both the real and imaginary parts. On the other hand, what is measurable is the magnetic field; hence no information is given about the scattered electric field, since it is interconnected with the corresponding magnetic field via Maxwell's equations.

The solution given here appears to be a good approximation at low frequencies and suitably describes the ellipsoidal shape behavior. The advantages of the formulation lie in the analytical expressions that yield closed-type compact forms, involving simple analytically known constant coefficients for any order of ellipsoidal harmonics introduced into the potentials. Consequently, any numerical evaluations of the fields could be very fast and can be achieved until the required convergence is obtained. This can be very useful for inverse schemes for localization and identification of buried objects at low frequencies, since the identification of an unknown scatterer can be better achieved as long as the field is clearly described. As a matter of fact, the need of higher-order harmonics at low frequencies certainly better fits future elaboration, in view of an inverse scheme.

\section{Conflicts of Interest}

The author declares that they have no conflicts of interest.

\section{References}

[1] G. Dassios and R. Kleinman, Low Frequency Scattering, Oxford University Press, Oxford, UK, 2000.

[2] G. Dassios, "Ellipsoidal Harmonics," in Theory and Applications, Cambridge University Press, Cambridge, UK, 2012.

[3] P. Moon and E. Spencer, Field Theory Handbook, Springer, Berlin, Germany, 1971.

[4] E. W. Hobson, The Theory of Spherical and Ellipsoidal Harmonics, Chelsea Publishing Company, New York, NY, USA, 1955.

[5] G. Perrusson, P. Vafeas, and D. Lesselier, "Low-frequency dipolar excitation of a perfect ellipsoidal conductor," Quarterly of Applied Mathematics, vol. 68, no. 3, pp. 513-536, 2010.

[6] G. Perrusson, P. Vafeas, I. K. Chatjigeorgiou, and D. Lesselier, "Low-frequency on-site identification of a highly conductive body buried in Earth from a model ellipsoid," IMA Journal of Applied Mathematics, vol. 80, no. 4, pp. 963-980, 2015.

[7] P. Vafeas, G. Perrusson, and D. Lesselier, "Low-frequency solution for a perfectly conducting sphere in a conductive medium with dipolar excitation," Progress in Electromagnetics Research, vol. 49, pp. 87-111, 2004.

[8] P. Vafeas, P. K. Papadopoulos, and D. Lesselier, "Electromagnetic low-frequency dipolar excitation of two metal spheres in a conductive medium," Journal of Applied Mathematics, vol. 2012, Article ID 628261, 2012.

[9] P. Vafeas, D. Lesselier, and F. Kariotou, "Estimates for the low-frequency electromagnetic fields scattered by two adjacent metal spheres in a lossless medium," Mathematical Methods in the Applied Sciences, vol. 38, no. 17, pp. 4210-4237, 2015.
[10] P. Vafeas, G. Perrusson, and D. Lesselier, "Low-frequency scattering from perfectly conducting spheroidal bodies in a conductive medium with magnetic dipole excitation," International Journal of Engineering Science, vol. 47, no. 3, pp. 372-390, 2009.

[11] S. J. Norton, W. A. SanFilipo, and I. J. Won, "Eddy-current and current-channeling response to spheroidal anomalies," IEEE Transactions on Geoscience and Remote Sensing, vol. 43, no. 10, pp. 2200-2209, 2005.

[12] P. Vafeas, P. K. Papadopoulos, P.-P. Ding, and D. Lesselier, "Mathematical and numerical analysis of low-frequency scattering from a PEC ring torus in a conductive medium," Applied Mathematical Modelling: Simulation and Computation for Engineering and Environmental Systems, vol. 40, no. 13-14, pp. 6477-6500, 2016.

[13] P. Vafeas, "Low-frequency electromagnetic scattering by a metal torus in a lossless medium with magnetic dipolar illumination," Mathematical Methods in the Applied Sciences, vol. 39, no. 14, pp. 4268-4292, 2016.

[14] H. Tortel, "Electromagnetic imaging of a three-dimensional perfectly conducting object using a boundary integral formulation," Inverse Problems, vol. 20, no. 2, pp. 385-398, 2004.

[15] Z. Xiong and A. C. Tripp, "Electromagnetic scattering of large structures in layered earths using integral equations," Radio Science, vol. 30, no. 4, pp. 921-929, 1995.

[16] J. Björkberg and G. Kristensson, "Three-dimensional subterranean target identification by use of optimization techniques," Progress in Electromagnetics Research, vol. 15, pp. 141-164, 1997.

[17] T. Yu and L. Carin, "Analysis of the electromagnetic inductive response of a void in a conducting-soil background," IEEE Transactions on Geoscience and Remote Sensing, vol. 38, no. 3, pp. 1320-1327, 2000.

[18] H. Huang and I. J. Won, "Detecting metal objects in magnetic environments using a broadband electromagnetic method," Geophysics, vol. 68, no. 6, pp. 1877-1887, 2003.

[19] X. Chen, K. O’Neill, B. E. Barrowes, T. M. Grzegorczyk, and J. A. Kong, "Application of a spheroidal-mode approach and a differential evolution algorithm for inversion of magnetoquasistatic data in UXO discrimination," Inverse Problems, vol. 20, no. 6, pp. 527-540, 2004.

[20] T. J. Cui, A. A. Aydiner, W. C. Chew, D. L. Wright, and D. V. Smith, "Three-dimensional imaging of buried objects in very lossy earth by inversion of VETEM data," IEEE Transactions on Geoscience and Remote Sensing, vol. 41, no. 10, pp. 2197-2210, 2003.

[21] H. Ammari and H. Kang, "Polarization and moment tensors: with applications to inverse problems and effective medium theor," in Applied Mathematical Sciences Series, vol. 162, Springer, New York, NY, USA, 2007. 


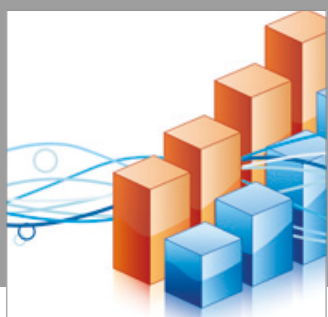

Advances in

Operations Research

vatersals

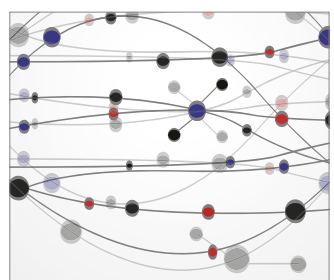

\section{The Scientific} World Journal
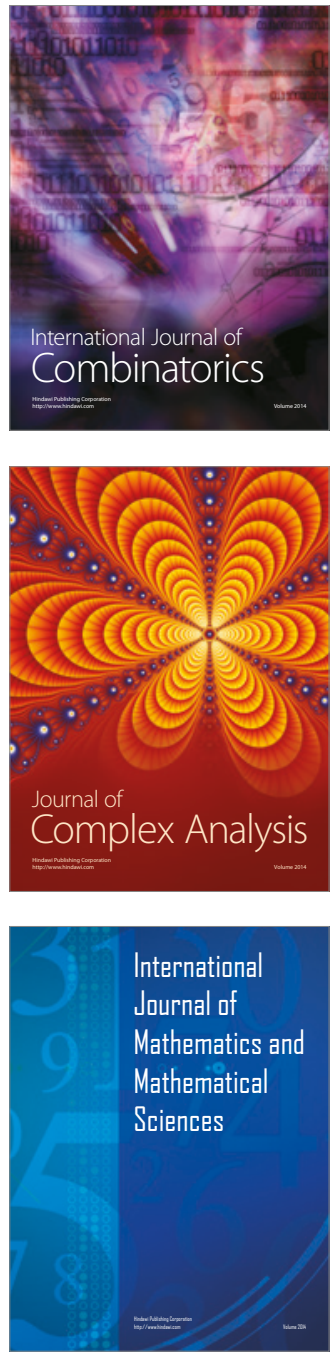
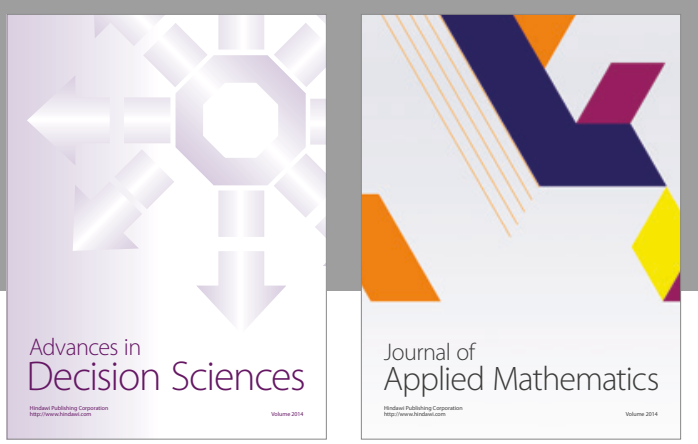

Algebra

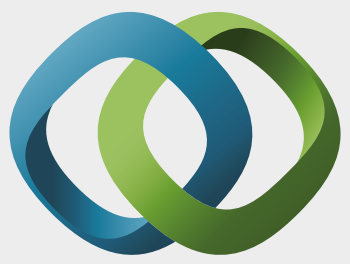

\section{Hindawi}

Submit your manuscripts at

https://www.hindawi.com
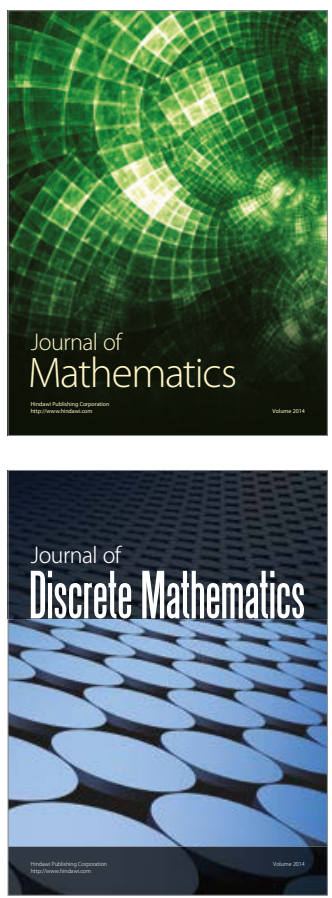

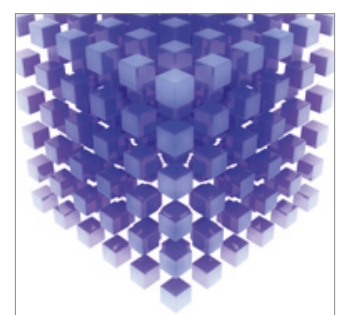

Mathematical Problems in Engineering
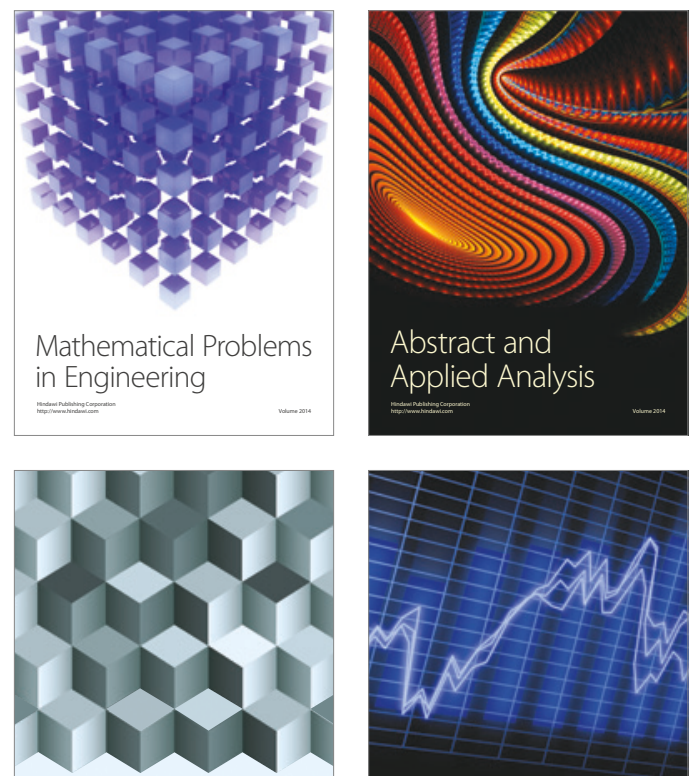

Journal of

Function Spaces

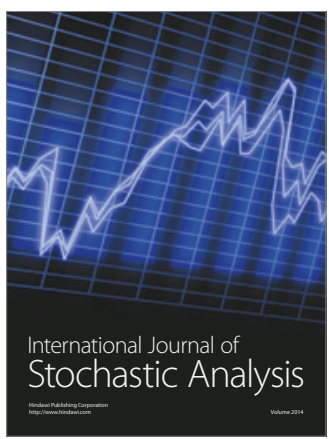

Probability and Statistics
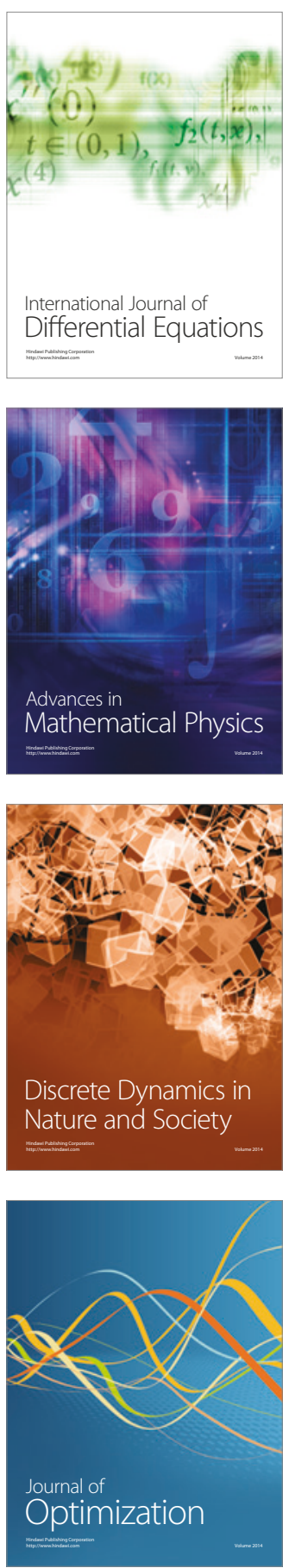